\title{
The Prediction and Observation of the 1997 July 18 Stellar Occultation by Triton: More Evidence for Distortion and Increasing Pressure in Triton's Atmosphere
}

\author{
J. L. Elliot, ${ }^{1,2}$ M. J. Person, and S. W. McDonald \\ Department of Earth, Atmospheric, and Planetary Sciences, Massachusetts Institute of Technology, 77 Massachusetts Avenue, \\ Cambridge, Massachusetts 02139-4307 \\ E-mail: jle@mit.edu
}

M. W. Buie, E. W. Dunham, R. L. Millis, R. A. Nye, C. B. Olkin, and L. H. Wasserman

Lowell Observatory, 1400 West Mars Hill Road, Flagstaff, Arizona 86001-4499

L. A. Young ${ }^{3}$

Center for Space Physics, Boston University, Boston, Massachusetts 02215

W. B. Hubbard and R. Hill

Lunar and Planetary Laboratory, University of Arizona, Tucson, Arizona 85721

H. J. Reitsema

Ball Aerospace, P.O. Box 1062, Boulder, Colorado 80306-1062

J. M. Pasachoff and T. H. McConnochie ${ }^{4}$

Astronomy Department, Williams College, Williamstown, Massachusetts 01267-2565

B. A. Babcock

Physics Department, Williams College, Williamstown, Massachusetts 01267-2565

R. C. Stone

U.S. Naval Observatory, Flagstaff Station, P.O. Box 1149, Flagstaff, Arizona 86002-1149

and

P. Francis

Mt. Stromlo Observatory, Private Bag, Weston Creek Post Office, Weston, ACT 2611, Australia

Received December 21, 1999; revised June 20, 2000

\footnotetext{
${ }^{1}$ Also at Lowell Observatory, 1400 West Mars Hill Road, Flagstaff, AZ 86001-4499.

${ }^{2}$ Also at Department of Physics, MIT, and guest observer, Cerro Tololo InterAmerican Observatory, National Optical Astronomy Observatories, operated by the Association of Universities for Research in Astronomy, Inc., under cooperative agreement with the National Science Foundation.

${ }^{3}$ Now at Southwest Research Institute, Suite 426, 1050 Walnut St., Boulder, CO 80302.

${ }^{4}$ Now at Astronomy Department, Coinell University, Ithaca, NY 14853.
}

A variety of CCD astrometric data was used to predict the location of the path for the occultation of the star we have denoted "Tr176" by Triton, which occurred on 1997 J uly 18, and was visible from locations in northern Australia and southern North America. A network of fixed and portable telescopes equipped with highspeed photometric equipment was set up to observe the event, with the following observational goals: (i) mapping the central flash (to 
establish the global shape of Triton's atmosphere at about 20-km altitude by modeling the detailed shape of the central flash), (ii) obtaining one or more light curves of high signal-to-noise ratio from a large telescope (to accurately determine the thermal structure of Triton's atmosphere), and (iii) obtaining light curves distributed across Triton's disk (to probe the thermal structure of Triton's atmosphere above different areas and to establish the shape of the atmosphere at about 100-km altitude by modeling the half-light surface). Although the large, fixed telescopes proved to be outside of the occultation shadow and observations with some of the portabletelescopes were foiled by clouds, light curves were successfully recorded from B rownsville, Texas, and C hillagoe, Queensland. These were combined with data from another group to determine the radius and shape of the half-light surface in Triton's atmosphere and the equivalent-isothermal temperatures at the sub-occultation latitudes on Triton. A circular solution for the half-light surface (projected into Triton's shadow) yielded a radius of $1439 \pm 10 \mathrm{~km}$. H owever, the data are indicative of a global shape more complex than a sphere. Such a figure is most likely caused by strong winds. Light-curve models corresponding to the best fitting circular and elliptical atmosphereswere fit to the data. Themean pressureat $1400-\mathrm{km}$ radius (48- $\mathrm{km}$ altitude) derived from all of the data was $2.23 \pm 0.28 \mu$ bar for the circular model and $2.45 \pm 0.32 \mu$ bar for the elliptical model. These values suggest a global pressure increase at this level since a previous Triton occultation in 1995 August. The mean equivalentisothermal temperatureat $1400 \mathrm{~km}$ was $43.6 \pm 3.7 \mathrm{~K}$ for the circular model and $42.0 \pm 3.6 \mathrm{~K}$ for the elliptical model. Within their (sometimes large) uncertainties, the equivalent-isothermal temperatures agree for all Triton latitudes probed. c) 2000 Academic Press

Key Words: Triton; occultations; atmospheres; structure.

\section{INTRODUCTION}

Several Voyager observations imply the presence of strong winds in Triton's lower atmosphere (Smith et al. 1989, Hansen et al. 1990). Surface winds to the northeast were inferred from the dust streaks, and cloud motions showed an easterly flow at an altitude of 1-3 km (Hansen et al. 1990). However, at the $\sim 8$-km altitude of the two detected plumes, the winds were blowing westward at an estimated velocity of 5-15 $\mathrm{m} \mathrm{s}^{-1}$ (Ingersoll 1990). Energy sources for winds include (i) the flow associated with maintaining vapor-pressure equilibrium of the major atmospheric constituent, $\mathrm{N}_{2}$, with surface frosts (Ingersoll 1990) and (ii) the uneven solar heating of Triton's variegated surface, where the frost is at a temperature of $38 \mathrm{~K}$ and the dark areas at a temperature of $\sim 57 \mathrm{~K}$ (Elliot et al. 2000).

From Earth-based observations, the presence of global winds can be inferred from the shape of a constant pressure level within the atmosphere, since Triton's rotation period and the direction of its pole are known (Seidelmann 1992). Probing Triton's atmosphere with a stellar occultation, one can determine its atmospheric figure with two complementary methods. The first is to establish a set of constant-pressure points by observing the occultation from a group of sites that span the full extent of Triton's atmosphere. One can then model these points with a circle or other atmospheric shape. The second method is to observe the occultation from one or more sites that passes near the center of Triton's shadow and model the structure of the "central flash" (Elliot et al. 1977) to learn the shape of the atmosphere, as was done for Titan from multi-station observations of its occultation of $28 \mathrm{Sgr}$ (Hubbard et al. 1993). The first method probes the shape of the atmosphere at altitudes of about $100 \mathrm{~km}$, while the second method probes the shape at about $20-\mathrm{km}$ altitude for Earth-based occultation observations of Triton's atmosphere.

Both methods have been applied to Triton occultation data for the star Tr148 (which occurred on August 14, 1995, McDonald and Elliot 1995). A circular solution with the immersion and emersion half-light times yielded a half-light radius (in Triton's shadow) of $1427.5 \pm 3.5 \mathrm{~km}$ (Olkin et al. 1997). An elliptical solution with the same data yielded a half-light semimajor axis of $1475.4 \pm 5.7 \mathrm{~km}$, but the ellipticity had a large error: $0.029 \pm 0.016$ (Olkin et al. 1997). On the other hand, the centralflash modeling yielded more precise results: $1432 \pm 2 \mathrm{~km}$ for the half-light semimajor axis and $0.018 \pm 0.003$ for the ellipticity (Elliot et al. 1997), which implies wind speeds near the speed of sound. Hence we wanted to record several light curves within the central region of Triton's occultation shadow in order to further investigate the structure of the central flash.

In addition to mapping the central flash, another motivation for further observation of stellar occultations by Triton is to look for changes in Triton's atmosphere with time. Various models have predicted that Triton's atmosphere should be expanding or collapsing, depending on the thermal properties of the surface (Hansen and Paige 1992, Spencer and Moore 1992).

The next occultation suitable for our goals was that of $\operatorname{Tr} 176$ (McDonald and Elliot 1995)_an $R=12.2$ magnitude star bright enough to yield good signal-to-noise with our $0.35-\mathrm{m}$ portable telescopes equipped with high-speed CCD photometers (Buie et al. 1993, Dunham 1995). This occultation was predicted to occur on 1997 July 18. Our early astrometry indicated that the event would be visible in eastern Australia, giving good deployment opportunities for these telescopes to map the central flash. This region also has some large, fixed telescopes that potentially could yield light curves of high quality for learning more about Triton's atmospheric structure.

Here we describe our pursuit and observation of Triton's occultation of Tr176. The plan for telescope deployment developed as our work on predicting the visibility zone for the occultation progressed. A notable aid to the prediction efforts was an appulse of Triton to Tr176 5 months prior to the occultation, which was observed over a period of a week from Cerro Tololo and Perth.

Following our observations of the Tr176 occultation, Elliot et al. (1998) reported Hubble Space Telescope observations of a subsequent occultation by Triton of the star $\operatorname{Tr} 180$, which occurred on 1997 November 4 (McDonald and Elliot 1995). They found that the surface pressure of Triton's atmosphere has increased since the time of Voyager, indicating a warming of the $\mathrm{N}_{2}$ surface frost. Also, a preliminary analysis of the $\operatorname{Tr} 176$ data set has indicated an increase of pressure in Triton's atmosphere 
since the Tr148 occultation (Sicardy et al. 1998). Here we describe a more extensive analysis of the $\operatorname{Tr} 176$ data set, in which we present our light curves for the occultation and-using both our data and that from the other group (Sicardy et al. 1998) perform a joint analysis that examines the size, shape, temperature, and pressure of Triton's atmosphere.

\section{OCCULTATION PREDICTIONS}

The occultation star (Tr176) was identified by McDonald and Elliot (1995) as part of a systematic search for occultation candidates for Triton carried out at MIT's George R. Wallace Jr. Astrophysical Observatory, located in Westford, Massachusetts. The astrometry used to identify the candidate placed the center of the occultation path $1800 \mathrm{~km}$ south of the geocenter, but with an error of $\pm 5000 \mathrm{~km}$. Additional astrometric observations obtained with the Flagstaff Astrometric Scanning Transit Telescope (FASTT, Stone et al. 1996) indicated a similar location for the shadow path, but with a smaller error. Accordingly, plans were made for observations and refining the predicted path of the event.

\section{Astrometric Observations}

An appulse of Triton to Tr176, which occurred on 1997 February $19-5$ months prior to the occultation-provided a good opportunity for refining the predicted path of the occultation. For 7 nights surrounding the appulse, for approximately $1 \mathrm{~h}$ each night, CCD frames were recorded with the STIS CCD on the Curtis-Schmidt telescope at Cerro Tololo, which has an entrance aperture of $0.6 \mathrm{~m}$ and a primary-mirror diameter of $0.9 \mathrm{~m}$ (Table I). The CCD was 2048 pixels square, with a focal-plane scale of $2.028 \mathrm{arcsec} /$ pixel that produced a field about $1.15^{\circ}$ on a side. A separate amplifier was used to read out each quadrant of the CCD, which could be accomplished in $40 \mathrm{~s}$. About half of the frames were recorded with a Kron-Cousins R filter and the other half with a custom filter that had a central wavelength of $720 \mathrm{~nm}$ and a passband of $50 \mathrm{~nm}$. The telescope tracked at a sidereal rate, producing CCD frames that we shall refer to as "stare frames" to distinguish them from "strip scans," for which the telescope is fixed and the rows of the CCD are clocked at the sidereal rate (Dunham et al. 1991). The integration times used were $10 \mathrm{~s}$ for the $\mathrm{R}$ filter and $40 \mathrm{~s}$ for the custom filter. Altogether, 280 frames suitable for astrometric analysis were recorded.

At Perth a CCD camera based on SNAPSHOT (Dunham et al. 1985, Dunham 1995) was mounted on the 0.6-m telescope and astrometric data were recorded for 8 nights (Table I). The $\mathrm{CCD}$ was a $2 \mathrm{~K}$ square Loral device, with a focal-plane scale of $0.73 \mathrm{arcsec} / \mathrm{pixel}$ that yielded a field $0.42^{\circ}$ on a side. The $\mathrm{R}$ and custom filter described above were also used, and the integration times for the exposures were 60 and $120 \mathrm{~s}$, respectively.

Other astrometric data sets were recorded with the $0.46-\mathrm{m}$ astrograph at Lowell Observatory and the 1.5-m Strand astrometric reflector at the U.S. Naval Observatory (USNO) in Flagstaff. Details of these data sets are in Table I. Astrometric data recorded with the Lowell astrograph differed from the other data sets in that the strip-scan mode was used (Dunham et al. 1991). Also, astrometric observations with the Fine Guidance System (FGS) aboard the Hubble Space Telescope were carried out on July 2, 1997, and these established that $\operatorname{Tr} 176$ was a single star, to a limit of no companion with $\Delta m \leq 2.5$ separated more than 50 mas from the brighter component. This allowed us to interpret the ground-based astrometric data in terms of a single star.

An important feature of all of the astrometric data used for the prediction is that each frame or strip scan contained the images of both Tr176 and Triton. Hence all astrometric reductions could be aimed at finding solutions that give accurate relative positions of these two objects; the accuracy of their individual positions in the J2000 system was not critical.

\section{Reduction at MIT}

The astrometric data were reduced at MIT by first correcting the raw CCD frames for bias and flat-field. Then the pixel centers of the astrometric reference stars, along with those for Triton and Tr176, were measured with the DAOPHOT package (Stetson 1987) in IRAF (Tody 1986). This routine established the point-spread function (PSF) for the frame by averaging the PSFs for several stars. Then it found the centers of other objects on the frame by fitting each with the frame PSF. Triton lies

TABLE I

Data A cquired for the Occultation Prediction

\begin{tabular}{|c|c|c|c|c|c|c|c|}
\hline CTIO & $0.6-0.9^{a}$ & STIS 2048 & $5120^{b}$ & 2.028 & $2 / 15-2 / 21$ & 280 & Elliot, Agner \\
\hline Lowell & $0.5^{c}$ & SNAPSHOT & 3200 & 0.844 & $5 / 29-7 / 17$ & 600 & Person, White, Motz \\
\hline Perth & 0.6 & SNAPSHOT & 145 & 0.73 & $2 / 13-2 / 20$ & 45 & Dunham \\
\hline USNO & 1.5 & Tek2K & 130 & 0.33 & $7 / 13-7 / 17$ & 200 & Bosh, Sickafoose \\
\hline
\end{tabular}

\footnotetext{
${ }^{a}$ The Curtis-Schmidt telescope at CTIO has an entrance aperture of $0.6 \mathrm{~m}$ and a primary diameter of $0.9 \mathrm{~m}$.

${ }^{b}$ Due to the large size of this data set, the frames were trimmed to the central $800 \mathrm{arcmin}^{2}$ before processing.

${ }^{c}$ Astrograph.
} 
TABLE II

Astrometric Analyses

\begin{tabular}{|c|c|c|c|c|c|c|c|}
\hline Analysis $^{a}$ & Filter & $\begin{array}{l}\text { Epoch } \\
\text { (1997) }\end{array}$ & $\begin{array}{l}\text { Reference } \\
\text { network }^{b}\end{array}$ & Centroid $^{c}$ & Reg. ${ }^{d}$ & $\begin{array}{l}\text { Ephemeris } \\
\text { correction }^{e}\end{array}$ & $\begin{array}{c}\text { Closest } \\
\text { approach (mas) }\end{array}$ \\
\hline U1 & $\mathrm{R}$ & $7 / 13-7 / 16$ & USNO [75] & DAOPHOT & Lin & Mean & $80 \pm 4$ \\
\hline U2 & $\mathrm{R}$ & $7 / 13-7 / 17$ & USNO [75] & DAOPHOT & Lin & Mean & $59 \pm 3$ \\
\hline U3 & $\mathrm{R}$ & $7 / 13-7 / 17$ & USNO [75] & DAOPHOT & Lin & Linear & $68 \pm 6$ \\
\hline U4 & $\mathrm{R}$ & $7 / 13-7 / 17$ & USNO [75] & DAOPHOT & Quad & Mean & $56 \pm 3$ \\
\hline U5 & $\mathrm{R}$ & $7 / 13-7 / 17$ & USNO [75] & DAOPHOT & Quad & Linear & $75 \pm 6$ \\
\hline $\mathrm{C} 1$ & 720 & $2 / 15-2 / 21$ & USNO [48] & Lorentzian & Lin & Mean & $62 \pm 16$ \\
\hline $\mathrm{C} 2$ & 720 & $2 / 15-2 / 21$ & USNO [48] & Lorentzian & Lin & Mean, $\Delta t$ & $61 \pm 25$ \\
\hline C3 & 720 & $2 / 15-2 / 21$ & USNO [48] & Lorentzian & Lin & Linear & $72 \pm 19$ \\
\hline $\mathrm{C} 4$ & 720 & $2 / 15-2 / 21$ & USNO [48] & Lorentzian & Lin & Linear, $\Delta t$ & $62 \pm 18$ \\
\hline C5 & $\mathrm{R}$ & $2 / 15-2 / 21$ & USNO [48] & Lorentzian & Lin & Mean & $43 \pm 18$ \\
\hline C6 & $\mathrm{R}$ & $2 / 15-2 / 21$ & USNO [48] & Lorentzian & Lin & Mean, $\Delta t$ & $74 \pm 23$ \\
\hline $\mathrm{C} 7$ & $\mathrm{R}$ & $2 / 15-2 / 21$ & USNO [48] & Lorentzian & Lin & Linear & $57 \pm 21$ \\
\hline C8 & $\mathrm{R}$ & $2 / 15-2 / 21$ & USNO [48] & Lorentzian & Lin & Linear, $\Delta t$ & $100 \pm 20$ \\
\hline C9 & 720 & $2 / 15-2 / 21$ & USNO [48] & DAOPHOT & Lin & Mean & $97 \pm 16$ \\
\hline $\mathrm{C} 10$ & 720 & $2 / 15-2 / 21$ & USNO [48] & DAOPHOT & Lin & Linear & $96 \pm 16$ \\
\hline C11 & $\mathrm{R}$ & $2 / 15-2 / 21$ & USNO [48] & DAOPHOT & Lin & Mean & $110 \pm 33$ \\
\hline C12 & $\mathrm{R}$ & $2 / 15-2 / 21$ & USNO [48] & DAOPHOT & Lin & Linear & $114 \pm 32$ \\
\hline \multirow{2}{*}{\multicolumn{7}{|c|}{$\begin{array}{l}\text { Predicted closest approach (unweighted average of } \mathrm{U} 2, \mathrm{C} 3 \text {, and C7) } \\
\text { Actual closest approach (from fit to the occultation data) }\end{array}$}} & $62.7 \pm 4.7$ \\
\hline & & & & & & & $74.9 \pm 0.4$ \\
\hline
\end{tabular}

a "U" denotes an analysis based on data from USNO, while "C" denotes an analysis based on data from CTIO. An unweighted average of analyses U2, C3, and C7 was used for the final prediction (see text).

$b$ "USNO" denotes a network of stars measured with FASTT.

${ }^{c}$ See text.

${ }^{d}$ The registration code "lin" denotes a 6-term linear transformation between pixel coordinates and celestial coordinates, while "quad" denotes a 12-term quadratic transformation.

${ }^{e}$ Here "mean" denotes a solution for only a mean offset between the measured positions for the star and Triton, while "linear" denotes a solution that allows a linear trend (with time) of the mean offset. For either case, " $\Delta t$ " allows for an offset in time for Triton relative to its ephemeris.

only $\sim 15$ arcsec from Neptune and, being nearly 5 magnitudes fainter, the determination of its center is affected by the wings of Neptune's image. Since establishing an accurate difference between the center of the images of $\operatorname{Tr} 176$ and Triton was critical to the astrometry, we tried an alternate method. First we fit the generalized Lorentzian model in Eq. (1) (see Eq. (1) of Bosh et al. 1992) to the image of $\operatorname{Tr} 176$ to establish the shape of the pointspread function. Then we used the two parameters that describe the shape of the PSF from this fit in a simultaneous fit of the images of Triton and Neptune for their centers and relative intensity. Saturated pixels in Neptune's image were omitted from the fit. In the subsequent steps of the reduction, these centers for Tr176 and Triton were used in place of the centers derived from DAOPHOT, although we retained the centers determined with DAOPHOT for the reference network stars. If time were available, a more rigorous application of this method would have been to (i) account for the different angular diameters of Neptune and Triton (which the DAOPHOT algorithm did not do either) and (ii) use the same PSF for the reference stars as we did for Triton and $\operatorname{Tr} 176$ (this would have made the absolute astrometry more accurate, in addition to the minimal requirement for the occultation prediction of accurate relative astrometry).

The pixel positions for the astrometric reference stars were then used to establish a transformation between the pixel coordi- nates and the $\mathrm{J} 2000$ astrometric system, in which we could solve either linearly for 6 transformation coefficients or quadratically for 12 transformation coefficients. Four different standard-star networks were available, but which was used depended on the field of view and homogeneity of the data set being analyzed. Here we refer to a data set as all of the data recorded with the same telescope, instrument, filter, and mode (strip scan or stare frame).

Table II presents a variety of reductions, each one different from the others with respect to either the data set used or the analysis procedures. Column 1 gives an identification of the analysis, with the first letter denoting the observatory where the data were recorded (Table I). Table II does not contain results of our analyses of the astrometric data from Perth or Lowell because these data sets yielded results inferior to those from the USNO and CTIO data sets and were not used in our final occultation prediction. In the case of the Lowell astrograph, the problem with the data was traced to an unexpectedly long time for the telescope to quit moving after being set in position to record a strip scan (unfortunately this cause was not identified until long after the occultation had occurred). The second column gives the filter used to record the data used in the analysis, and the fourth gives the source of the astrometric network. The reference stars were measured with FASTT at USNO (Stone et al. 1996). The number 
in square brackets is the number of stars in the network that were used in a particular analysis, and this number was chosen as the maximum set of stars that appear on all frames of a data set.

The fifth column gives the method for finding the centroids for a particular analysis, where "DAOPHOT" means that it generated all centroids. "Lorentzian" means that the generalized Lorentzian fit (as mentioned above) was used for $\operatorname{Tr} 176$ and Triton (with DAOPHOT being used for finding the centroids of the reference stars). The method for registering the pixel coordinates of the reference stars to the $\mathbf{J} 2000$ system is given in the sixth column, where "lin" denotes the linear registration and "quad" the quadratic registration.

In the penultimate column of Table II we present the method used to extrapolate the correction to Triton's ephemeris, as determined by the astrometric reductions, to the time of the occultation in order to establish a prediction. In discussing this extrapolation it is conceptually easier to assume that all the error is in Triton's ephemeris. Although this case is highly unlikely, it makes an insignificant difference to the occultation prediction, since we are concerned only with the relative position of $\operatorname{Tr} 176$ and Triton. One approach is to assume that there is a mean offset in RA and Dec between Triton's true position and its ephemeris, and we denote these solutions by "mean." Another approach is to allow a linear drift of this offset with time, which basically corresponds to a small rotation between the J2000 system as defined by the network of reference stars (each of which have positional errors) and the J2000 system as defined by Triton's ephemeris. In addition to solving for a mean or linear offset, one can also allow an offset in time between Triton's position and its ephemeris, which would appear if there were a longitudinal error in its ephemeris. We denote the inclusion of this effect by " $\Delta t$ " in the seventh column of Table II.

Finally, in the last column of Table II, we give the corrected "closest approach," which is defined as the minimum distance between the center of Triton's shadow to a hypothetical observer at the center of the Earth. The error bars are formal errors that are based on the internal consistency of each analysis. For comparison, the angle subtended by Triton's atmospheric half-light radius is about 70 mas.

Examining the predicted closest approach distances for Table II we see that their scatter is more than their formal errors. For the USNO data we felt the most reliable result was likely to be from the U2 analysis because (i) it included the entire USNO data set, (ii) quadratic registration was not warranted, and (iii) there was no significant linear trend in the extrapolation for this data set.

The CTIO data were recorded at extremely high airmass, and we had a priori reasons for preferring the 720 -nm data over that from the R filter: (i) the Neptune background near Triton was less, (ii) the refractive dispersion over the filter bandwidth was less, and (iii) the longer exposures would have averaged out the differential refraction between the objects on the frame within different isoplanatic patches. In the end, however, we decided to accept the 720-nm filter and R-filter data on an equal basis, because we wanted to avoid injecting more "judgment calls" into the occultation prediction than were absolutely necessary. We selected analyses C3 (720-nm-filter data) and C7 (R-filter data) because (i) our model routine yielded more consistent results for the centroiding for Triton (in close proximity to Neptune) than we achieved with DAOPHOT, (ii) there was no significant time offset of Triton from its ephemeris, and (iii) a significant linear trend appeared in the extrapolation for this data set.

Then we established our best value for the predicted closest approach distance as an unweighted average of these three results (U2, C3, and C7), since it was clear that the formal errors are not good indicators of the real errors. The resulting closest approach distance is $62.7 \pm 4.7$ mas, where the error was calculated from the scatter of the three values. Based on this astrometric solution, predictions for the sites of all known observers were posted on the MIT website (http://occult.mit.edu) $10 \mathrm{~h}$ prior to the event. In Table III we have given the predictions for the geocenter and our four portable telescopes based on this astrometric result. The predicted shadow path is illustrated in Fig. 1, where the three dashed lines represent the predicted path of the northern limb, centerline, and southern limb.

Although the astrometric analysis just described was used for our final prediction, during the 2 months prior to the event

\section{TABLE III}

Final Prediction and Comparison with Observed Results

\begin{tabular}{|c|c|c|c|}
\hline Station & $\begin{array}{l}\text { Closest approach } \\
(\mathrm{km})\end{array}$ & $\begin{array}{l}\text { Event midtime }^{a} \\
\text { (UTC) }\end{array}$ & $\begin{array}{c}\text { Chord length } \\
(\mathrm{km})\end{array}$ \\
\hline \multicolumn{4}{|l|}{ Brownsville } \\
\hline Predicted & $826 \pm 85$ & $10: 10: 06 \pm 4$ & $2339 \pm 120$ \\
\hline Observed & & $10: 10: 25 \pm 0.2$ & $2640 \pm 10$ \\
\hline Circular fit ${ }^{c}$ & $569 \pm 7$ & $10: 10: 28 \pm 0.7$ & $2644 \pm 8$ \\
\hline Elliptical fit ${ }^{d}$ & $564 \pm 5$ & $10: 10: 26 \pm 0.5$ & $2642 \pm 6$ \\
\hline \multicolumn{4}{|l|}{ Chillagoe } \\
\hline Predicted & $-265 \pm 85$ & $10: 17: 33 \pm 4$ & $2815 \pm 32$ \\
\hline Observed & & $10: 17: 53 \pm 0.4$ & $2679 \pm 14$ \\
\hline Circular fit ${ }^{c}$ & $-521 \pm 7$ & $10: 17: 54 \pm 0.7$ & $2683 \pm 7$ \\
\hline Elliptical fit ${ }^{d}$ & $-524 \pm 5$ & $10: 10: 54 \pm 0.5$ & $2676 \pm 5$ \\
\hline \multicolumn{4}{|l|}{ Coen } \\
\hline Predicted & $10 \pm 85$ & $10: 17: 34 \pm 4$ & $2862 \pm 1$ \\
\hline Circular $\mathrm{fit}^{c}$ & $-246 \pm 7$ & $10: 17: 53 \pm 0.8$ & $2836 \pm 3$ \\
\hline Elliptical fit ${ }^{d}$ & $-251 \pm 8$ & $10: 17: 53 \pm 0.5$ & $2830 \pm 2$ \\
\hline \multicolumn{4}{|l|}{ Geocentric } \\
\hline Predicted $^{b}$ & $-1323 \pm 85$ & $10: 13: 50 \pm 4$ & $1133 \pm 155$ \\
\hline Circular fit ${ }^{c}$ & $-1580 \pm 7$ & $10: 14: 08 \pm 0.7$ & $e$ \\
\hline Elliptical fit ${ }^{d}$ & $-1585 \pm 5$ & $10: 14: 06 \pm 0.5$ & $e$ \\
\hline \multicolumn{4}{|l|}{ Troncones } \\
\hline Predicted & $211 \pm 85$ & $10: 10: 16 \pm 4$ & $2864 \pm 25$ \\
\hline Circular fit $^{c}$ & $-45 \pm 7$ & $10: 10: 36 \pm 0.8$ & $2877 \pm 1$ \\
\hline Elliptical fit ${ }^{d}$ & $-50 \pm 1$ & $10: 10: 36 \pm 0.3$ & $2872 \pm 1$ \\
\hline
\end{tabular}

\footnotetext{
${ }^{a}$ After $199707180 \mathrm{~h} \mathrm{UT}$.

${ }^{b}$ Unweighted mean of solutions C3, C7, and U2 from Table II.

${ }^{c}$ Calculated from astrometric solution Fit No. 2 in Table VI.

${ }^{d}$ Calculated from astrometric solution Fit No. 6 in Table VI.

${ }^{e}$ Triton's shadow did not pass over the geocenter.
} 


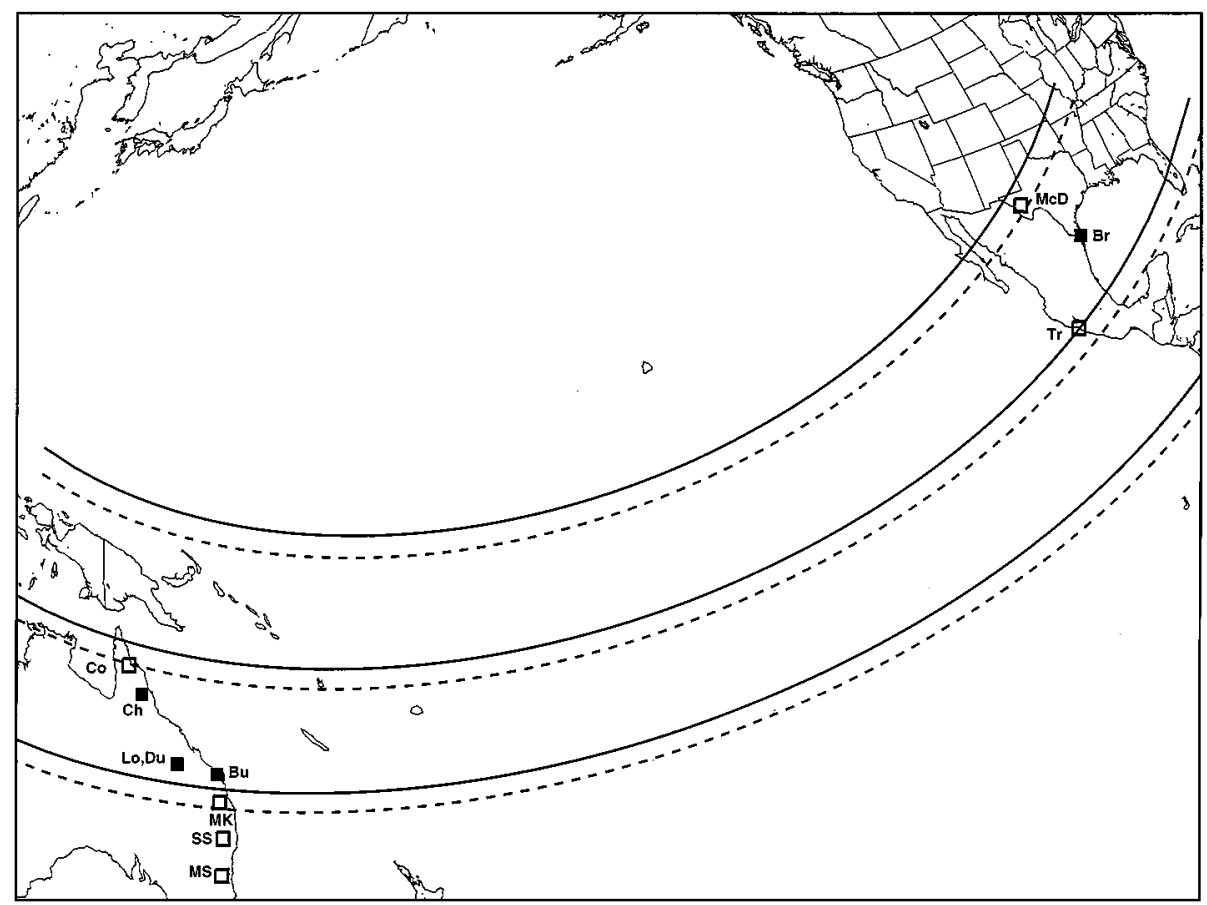

FIG. 1. Predicted shadow path and worldwide observing sites. Dotted lines show the final predicted shadow path referenced in Table III. Solid lines show the astrometric solution calculated from observed chords. Solid squares indicate sites acquired data used in this analysis, and open squares show sites where our consortium's observations were unsuccessful, either due to clouds or being outside Triton's shadow as it crossed the Earth. The stations are labeled as follows: Br, Brownsville; Bu, Bundaberg; Ch, Chillagoe; Co, Coen; Du, Ducabrook; Lo, Lochington; McD, McDonald; MK, Mount Kent; MS, Mount Stromlo; SS, Siding Spring; Tr, Tracones.

copious tests were run with a variety of methods for each stage of the prediction calculations. For example, in addition to the procedures described above, different choices of the referencestar network were used to compare results from using the most accurate positions available (Hipparcos) versus those having a larger number of stars (USNO A1.0). Both JPLs DE-403 and DE-118 ephemerides were used. Also, solutions were found by registering the pixel positions to celestial coordinates, with and without the tangent-plane projection (Smart 1977).

\section{Reduction at Lowell}

Our desire to get at least two ground stations in a location that would sample the central flash required an extremely accurate prediction, and we knew that systematic errors would be as important as random errors. Therefore, a prediction effort at Lowell Observatory was undertaken in parallel with the MIT efforts. This effort used a completely separate software pipeline and at times a different algorithmic approach. At Lowell (as at MIT) we experimented with different methods of centroiding, different reference-star networks, and different terms in the equations used to register the astrometric frames to the reference-star network. Since the time available for this work prior to deployment of our portable telescopes was limited, these reductions were not carried out as extensively as we would have liked. Furthermore, as will be shown later, the results contain yet unidentified systematic errors, which we had hoped to identify prior to publication of these results. However, with the objective of promoting further understanding of the astrometric methods that we used to predict this occultation, we shall present our results in the time order that they were generated, offering what insights that we can.

Figure 2 shows a graphical summary of the results in terms of right ascension and declination. By applying this offset to the ephemeris of Triton, we can predict the shadow path of the event. (For comparison, note that the MIT results in Table II are presented in terms of the offset and its error perpendicular to the motion of Triton's shadow.) The zero-point of Fig. 2 is set by the astrometric solution inferred from the occultation light curves (to be discussed later) and represents the truth sought in the prediction (solid line). Overlain on the plot is the size of Triton (dashed lines) and the rough size of the central flash region (dotted lines). Shown in this fashion, a correct prediction is one that includes zero within its uncertainty. However, to be useful, the uncertainty of the prediction must be less than the size of the central flash region.

The main astrometric data sets used for the predictions plotted in Fig. 2 are as follows: (i) stars, Perth; (ii) triangles, CTIO; (iii) squares, USNO. Very few predictions were computed with the Perth data, as it was quickly apparent that the internal scatter in those data was just too large to be useful. Triangles (up through prediction 41) mark predictions based on the CTIO data. Evolution of the CTIO-based predictions involved changes in software, reference catalogs, and position extraction techniques. In 


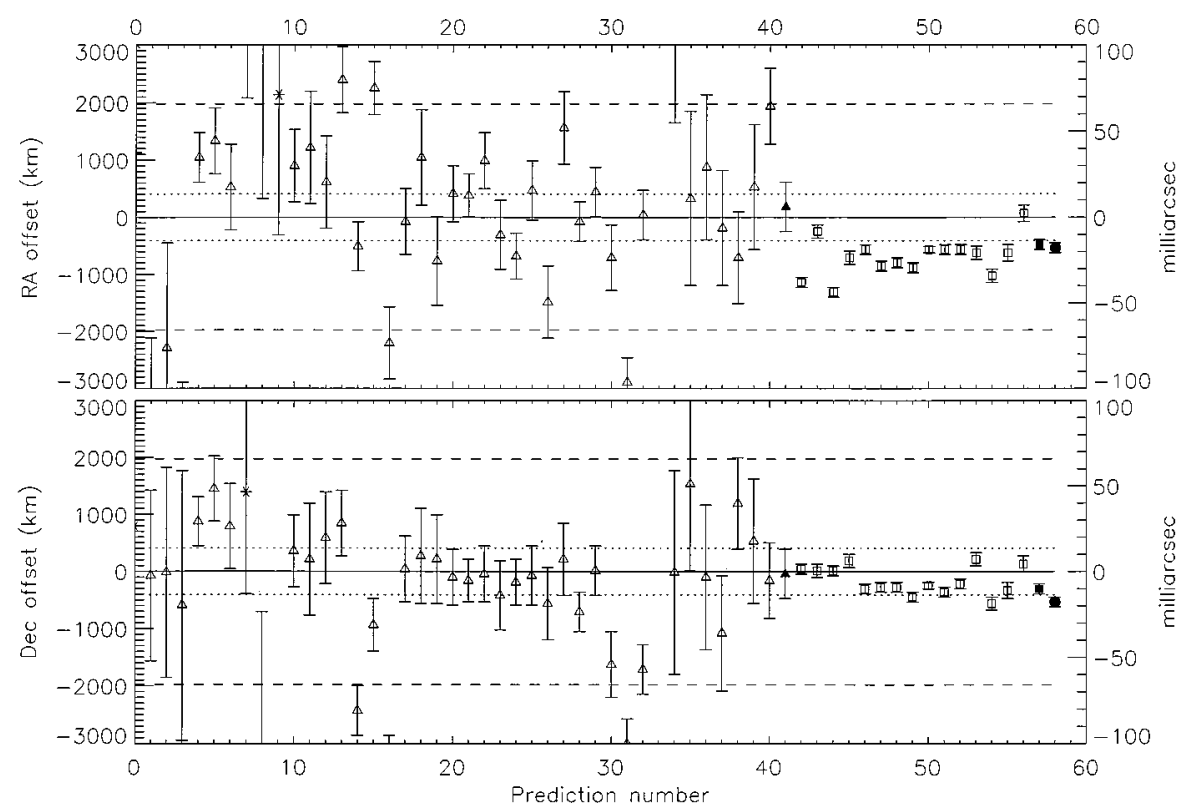

FIG. 2. Lowell predictions relative to the path of the occultation. The upper panel shows the offset in right ascension plotted versus prediction number, which is roughly proportional to time. The lower panel shows the offset in declination. Generally, the higher the prediction number, the better the prediction should be. The offset is shown in kilometers (projected onto the Earth at the Earth-Triton distance) on the left and in milliarcseconds on the right. The size of Triton's atmosphere at the half-light level is indicated by the dashed lines, and the rough size of the central-flash region is shown by the dotted lines. The zero-point of this figure is set by the geometry inferred from the occultation light curves (to be discussed later) and represents the truth sought in the prediction (solid line). Asterisks represent predictions based on the Perth data. Triangles represent predictions based on the CTIO data. The evolution of the CTIO-based predictions was caused by changes in software, reference catalogs, and position extraction techniques. Squares represent predictions based solely on the data taken at USNO (Table I), which differ from the "truth" by much more than their formal errors due to one or more unknown systematic errors. The final prediction from MIT is shown by the last point in each panel (solid circle). See text for a discussion of these predictions.

these Lowell reductions it was found that the declination direction could be registered to the network of reference stars in a purely linear fashion. However, the right ascension direction required the addition of quadratic plate constants to achieve a good fit. All of the predictions shown by the open triangles through number 20 were computed with the aperture-based, center-oflight measurements of all stars and Triton. The details of this positional extraction method are discussed in Buie and Bus (1992). Predictions 21-32 were based on the DAOPHOT positions as measured as part of the MIT effort. While it may not be obvious from this plot, the PSF-based positions were marginally better than the aperture sum positions.

Predictions 42-57, represented by the squares in Fig. 2, were based solely on the data taken at USNO (Table I). These predictions were computed with a mix of aperture summed-centroids and DAOPHOT centroids. For these data, the choice of position extraction method did not make any difference. Note that the final MIT prediction (shown in Fig. 1 and Table III) is plotted as the last prediction in Fig. 2 as a solid circle.

There are two interesting subsets of predictions shown in Fig. 2. Members of the first subset are 34-40. These are predictions based on each night of CTIO data, reduced individually. The filled triangle (prediction 41) that follows is the average of all but the first night of CTIO data. These predictions are drawn from only the 720-nm filter observations (i.e., R-band data were excluded). Note that the scatter between nights is consistent with the uncertainties and the grand average is in fact a "correct" solution. Prediction 41 was considered the best possible prediction at Lowell from the CTIO data after nearly 2 months of painstaking work. In hindsight, this prediction met all of our requirements for a valid and useful prediction. The deployment of the ground stations would have been accurate based on only this prediction.

However, an occultation prediction based on a pre-occultation appulse had been done only once before (by Franz and Wasserman to predict an occultation by Uranus in 1977; see IAU Circulars 3038 and 3040). This fact combined with the tight astrometric requirements lead us to pursue observations in the days just prior to the event. The prediction subset 53-56 shows the single-night predictions and the final averages (filled square). In this case, note that the scatter between nights is much larger than the error bars from each night alone. Also note that the USNO data led to predictions that were systematically offset from the truth by more than their formal errors.

It may be that the difference in the noise behavior between the CTIO and USNO data is due to small but systematic rotations in the reference-star grid. Both reductions were supported by data collected by FASTT, and the random and systematic errors are probably at the same level. CTIO and USNO differed in both field of view and pixel scale. CTIO covered a much larger field but at a coarser pixel scale. The coarser pixel scale is 
probably the cause of the higher random errors. However, the larger field would minimize any systematic rotation errors in the plate solutions.

\section{Summary of Prediction Methodology}

Looking back on the prediction effort there were a number of lessons learned and a few more questions raised. The best predictions come from a process that blends good data and software reductions with a substantial level of human judgment. Blindly taking data and reducing it by "turning the crank" does not insure the best answer. Unfortunately, adding in human judgment is a process that requires considerable time. In the case where data are taken in the days just prior to the event, there is hardly enough time to add enough judgment. From this standpoint, our technique of using a pre-occultation appulse to generate a prediction should be the technique of choice as long as the orbit of the object is very well known. Despite making considerable progress in our prediction techniques, we still face the challenge of understanding the true errors in our predictions, since the scatter of the results (both in Table II and Fig. 2) is greater than the formal errors based on the internal consistency of each analysis.

Although a complete description of all the work carried out to test the consistency of our astrometric reductions is well beyond the scope of this paper, we summarize here the factors that we found to be important for accurate occultation-prediction astrometry for Triton with CCD data: (1) both the occultation star and Triton should be recorded on the same frame, which can be done either near an appulse (for which one can interpolate to find the closest approach distance of Triton to the star) or immediately prior to the occultation (for which one must extrapolate to find the closest-approach distance); (2) an accurate, self-consistent set of astrometric reference stars is essential; (3) a set of reference stars common to all frames used in a re- duction should be selected (e.g., the set of reference stars should not change from frame to frame); (4) the reference stars should surround the candidate star and all positions of Triton, so that no extrapolation outside the reference network is ever necessary to establish Triton positions; (5) a limiting factor in determining an accurate center for the Triton PSF is the model used for the wings of the Neptune PSF; (6) the Triton and Neptune images should not be used to construct the average PSF for each frame; and (7) the longer the exposure time for astrometric frames, the more that astrometric errors caused by differential refraction between objects in different isoplanatic patches are averaged out.

\section{OBSE RVATIONS}

Our observational goals for this event were to (i) map the central flash with a set of portable telescopes (to establish the ellipticity of Triton's atmosphere at about 20-km altitude by modeling the detailed shape of the central flash), (ii) obtain one or more light curves of high signal-to-noise ratio from a large telescope (to accurately determine the thermal structure of Triton's atmosphere), and (iii) obtain light curves distributed across Triton's disk (to probe the thermal structure of Triton's atmosphere above different areas and establish the shape of the atmosphere at about $100-\mathrm{km}$ altitude by modeling the half-light surface). Hence our observational plan employed two approaches: to use large telescopes that might be within the occultation path and to deploy four portable telescopes in an evenly spaced grid, spanning the central flash region near the center of the shadow. The sites from which our consortium attempted observations are summarized in Table IV. Here we list for each site the telescope aperture, instrument, instrumental parameters, and observers.

Although we initially considered observations from Mauna Kea, early on it became clear that the occultation path would

\section{TABLE IV}

Occultation Observing Sites ${ }^{a}$

\begin{tabular}{|c|c|c|c|c|c|c|}
\hline Site & $\begin{array}{c}\text { Telescope } \\
\text { aperture (m) }\end{array}$ & Instrument & $\begin{array}{l}\text { Image scale } \\
(\operatorname{arcsec} / \mathrm{pix})\end{array}$ & $\begin{array}{l}\text { Subframe } \\
(\text { row } \times \text { col })\end{array}$ & $\begin{array}{l}\text { Integration } \\
\text { time (s) }\end{array}$ & Observers \\
\hline Chillagoe & 0.35 & PCCD & 1.2 & $81 \times 91$ & 0.5 & Elliot, Person \\
\hline $\operatorname{Coen}^{b}$ & 0.35 & PCCD & 1.2 & $80 \times 90$ & 0.5 & Dunham, Young \\
\hline McDonald & 0.9 & 2-ch phot. ${ }^{c}$ & $c$ & $c$ & $c$ & Wasserman, Nye \\
\hline Siding Spring & 2.3 & CASPIR & 0.5 & $f$ & $f$ & Francis \\
\hline Troncones $^{b}$ & 0.35 & PCCD & 1.2 & $80 \times 90$ & 0.5 & Buie \\
\hline
\end{tabular}

\footnotetext{
${ }^{a}$ Sites for the IOTA group are given in Sicardy et al. (1998).

${ }^{b}$ Coordinates (east longitude, latitude, altitude in meters) for the telescope south of Coen were (143 $\left.21^{\prime} 48^{\prime \prime},-14^{\circ} 24^{\prime} 00^{\prime \prime}, 200\right)$; at Mt. Kent, $\left(151^{\circ} 51^{\prime} 32^{\prime \prime},-27^{\circ} 47^{\prime} 58^{\prime \prime}, 678\right)$, and at Troncones, (-101 $\left.43^{\prime} 13.2^{\prime \prime},+17^{\circ} 46^{\prime} 43.7^{\prime \prime}, 3\right)$.

${ }^{c}$ At McDonald and Mount Stromlo, aperture photometers were used.

${ }^{d}$ The Williams College Fast Coronal Imager.

${ }^{e}$ This is the size of the subframe in binned pixels, each of which is $2 \times 2$ actual pixels on the detector.

${ }^{f}$ The Cryogenic Array Spectrometer/Imager (CASPIR) was used with a J filter $(1.3 \mu \mathrm{m})$. The telescope was set to a slow slew so the image of Triton and Tr176 trailed across the $256 \times 256$ InSb detector in $180 \mathrm{~s}$, which yielded an effective time resolution of slightly less than $1 \mathrm{~s}$.
} 
pass well south of Hawaii, so we concentrated on setting up observations on the 1.7-m telescope at Mount Stromlo and the 2.3-m telescope at Siding Spring. Only close to the time of the event did it become a good possibility that the occultation path would pass over McDonald, where we were able to use the $0.9-\mathrm{m}$ telescope on short notice. Conditions were poor at McDonald, with variable cirrus reflecting the bright moonlight, and no useful data were recorded. Clear weather prevailed at Mount Stromlo and Mt. Kent, but these sites proved to be south of the occultation path (Fig. 1).

In addition to these observations from fixed telescopes, four portable telescopes equipped with high-speed, portable CCD photometers (PCCD, Buie et al. 1993, Dunham 1995) were planned to be deployed in a grid spanning the predicted centralflash region, with an approximate spacing of $0.01 \mathrm{arcsec}$, (corresponding to $211 \mathrm{~km}$ at the Earth-Triton distance). This strategy was set up on the premise that our prediction would be in error by no more than \pm 0.02 arcsec (which corresponds to $\pm 422 \mathrm{~km}$ at the Earth-Triton distance). With this grid in place and if the shadow would pass where predicted, the two central stations would be about $106 \mathrm{~km}$ from the center of the shadow, and the outer two stations would be about $317 \mathrm{~km}$ from the center. If the prediction error were 0.02 arcsec, however, one of the boundary stations would be $106 \mathrm{~km}$ from the center, with each other station $211 \mathrm{~km}$ further from the center than its more central neighbor.

About 2 weeks prior to the event, when we had to decide what portable equipment to ship to Australia, the prediction indicated that Triton's shadow would straddle northern Queensland and southern North America, with some overlap. Since these regions are on opposite sides of the Earth, it would not be possible to rapidly shift our portable stations between the two zones near the time of the occultation. Hence we deployed two of our portable stations in Australia and two in North America.

Due to weather prospects and logistical considerations, we did not achieve the precise locations indicated by our planned grid. The initial strategy called for the LPL equipment to be lo- cated $317 \mathrm{~km}$ north of the predicted centerline in central Mexico. However, extremely poor weather prospects there led to a lastminute decision to shift the LPL observing site to Brownsville, Texas, where clear skies were forecast, although there was no prospect of observing a Triton central flash at that site. The MIT station and one of the Lowell stations were deployed in northern Queensland, Australia — at Chillagoe and near Coen, respectively-while the other Lowell station was deployed at Troncones, Mexico. The stations were deployed, based on the prediction 2 days before the event, which indicated a shadow path that was about $70 \mathrm{~km}$ north of the final prediction (Fig. 1). The locations of our stations, relative to the ideal locations set by the deployment prediction, for our portable stations were (from north to south along the grid): Brownsville, $331 \mathrm{~km}$ north; Troncones, $35 \mathrm{~km}$ north; Coen, $46 \mathrm{~km}$ north; and Chillagoe, $18 \mathrm{~km}$ south.

The four portable stations were in place for test observations on the night before the event. No filters were used in order to maximize throughput, and the sub-frame sizes used for the observations (Table IV) were set to include a reference star of similar brightness as Tr176 that lies less than an arc minute southwest of it. Observing conditions were excellent in Brownsville, with photometric skies and sub-arcsecond (occasionally diffractionlimited) seeing. The telescope was located at a site overlooking a resaca (oxbow lake) of the Rio Grande. These circumstances apparently helped to produce superb image quality despite a sea-level site and high airmass. Flat fields were obtained on the dawn sky immediately following the occultation. At Chillagoe, skies were clear, but an intermittent light breeze caused momentary vibrations of the telescope. Unfortunately, attempted observations from Coen and Troncones were clouded out.

Additional occultation light curves from three sites in Australia were recorded by the International Occultation Timing Association (IOTA, Sicardy et al. 1998). In Table V we present the coordinates (in WGS84 coordinates, as determined with the Global Positioning System, (GPS)) for all stations where data were recorded that are used in our analyses.

TABLE V

Observatory Coordinates ${ }^{a}$ and Half-Light Times

\begin{tabular}{|c|c|c|c|c|c|c|}
\hline Site $^{b}$ & $\begin{array}{l}\text { East longitude } \\
\qquad\left({ }^{\circ},{ }^{\prime} \prime\right)\end{array}$ & $\begin{array}{l}\text { Latitude } \\
\left({ }^{\circ}, \prime^{\prime \prime}\right)\end{array}$ & $\begin{array}{l}\text { Altitude } \\
\quad(\mathrm{m})\end{array}$ & $\begin{array}{c}\text { Time of half-light }{ }^{c} \\
\text { (UTC) }\end{array}$ & $\begin{array}{c}f_{\mathrm{pr}} \\
(\mathrm{km})\end{array}$ & $\begin{array}{c}g_{\mathrm{pr}} \\
(\mathrm{km})\end{array}$ \\
\hline Brownsville, I & -973211.3 & +255840.9 & -0.9 & $10: 09: 32.22 \pm 0.34$ & -32392 & -4599 \\
\hline Brownsville, E & & & & $10: 11: 18.54 \pm 0.34$ & -29901 & -3725 \\
\hline Bundaberg, I & 1522235.4 & -245635.7 & 10 & $10: 17: 10.97 \pm 0.40$ & -31075 & -6094 \\
\hline Bundaberg, E & & & & $10: 18: 04.49 \pm 0.40$ & -29822 & -5641 \\
\hline Chillagoe, I & 1443136.5 & -170857.4 & 368.6 & $10: 16: 59.29 \pm 0.67$ & -32006 & -5633 \\
\hline Chillagoe, E & & & & $10: 18: 47.05 \pm 0.24$ & -29487 & -4719 \\
\hline Ducabrook, I & 1472640.0 & -235355.0 & 320 & $10: 17: 17.94 \pm 0.49$ & -31202 & -6076 \\
\hline Ducabrook, E & & & & $10: 18: 19.72 \pm 0.49$ & -29757 & -5553 \\
\hline Lochington, I & 1473124.8 & -235642.5 & 270 & $10: 17: 17.73 \pm 0.52$ & -31202 & -6080 \\
\hline Lochington, E & & & & $10: 18: 19.79 \pm 0.52$ & -29750 & -5555 \\
\hline
\end{tabular}

${ }^{a}$ Geodetic, WGS84.

$b$ "I" denotes immersion and "E" denotes emersion. The coordinates are the same for both events.

${ }^{c}$ After 19970718 at $00: 00 \mathrm{~h}$. 


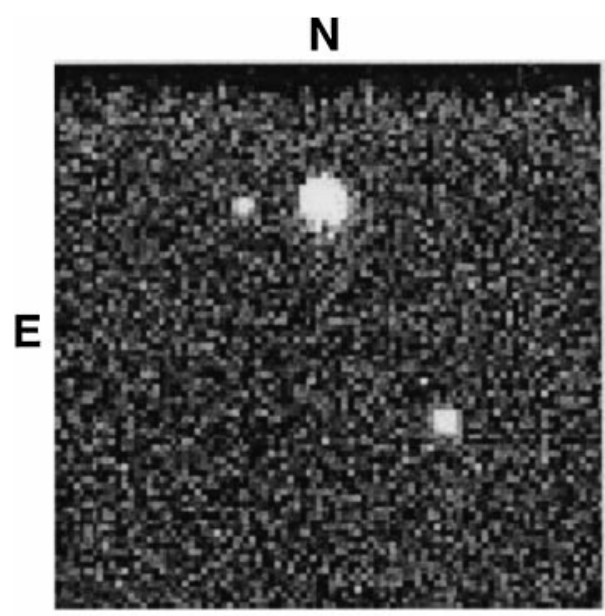

Brownsville

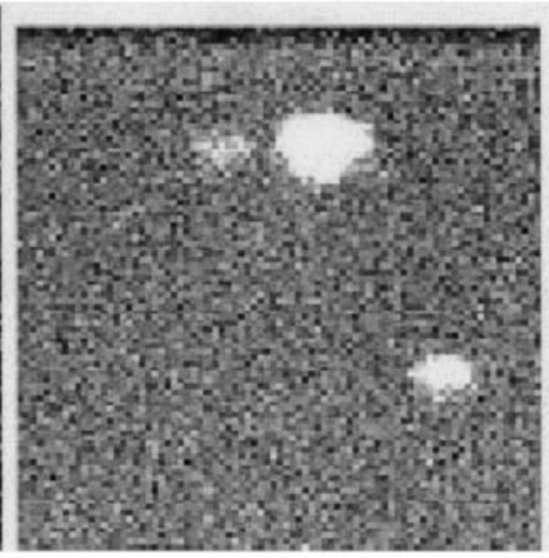

Chillagoe with wind shake

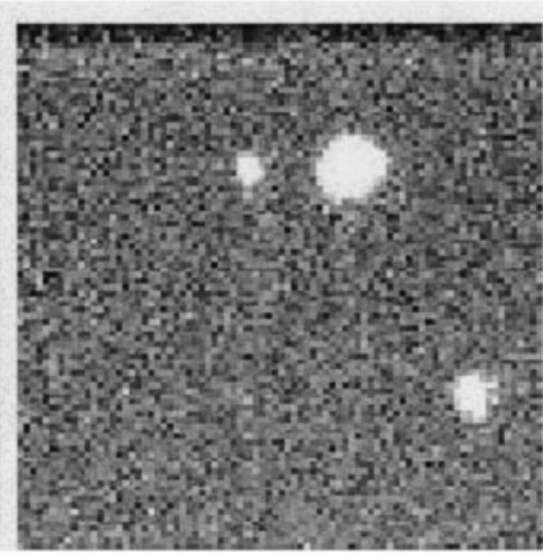

Chillagoe

FIG. 3. Three sample images are displayed. The first is a typical image from the Brownsville image cube. The second image shows the wind shake that led to exclusion of about half of the Chillagoe data points and is one if the best images of those discarded. The third is a representative image from the usable portions of the Chillagoe image cube. All three images have exposure times of $0.5 \mathrm{~s}$, as given in Table IV, with north at the top and East on the left. The large body in the upper center of the frames is Neptune, with the blended Tr176/Triton just to its left. The image in the lower right is a reference star.

\section{LIGHT CURVES}

For Brownsville, data cubes consisted of 1600 frames each on a 90-pixel by 90-pixel CCD field (1 pixel $=1.2$ arcsec) which included Triton, $\operatorname{Tr} 176$, Neptune, and a reference star (Fig. 3). The frames were recorded every $0.5 \mathrm{~s}$. Additional data cubes were obtained $1.5 \mathrm{~h}$ prior to the event to permit separate photometry of Triton and Tr176. The cube containing the occultation was started at 10:02:14 UTC as noted manually on the observing log and confirmed by the header on the data file. Throughout the night, the GPS system maintained lock and there was never any indication of any malfunction in the timing chain used to trigger the frame transfers.

The reduced light curve (Fig. 4) was obtained through the use of the reference star and the PSF method described below. First, all image cubes were bias-subtracted and flat-fielded. Then, the reference star, located about 32 pixels west and 36 pixels south of $\operatorname{Tr} 176$, was fitted with a Gaussian profile. The flux from the reference star was approximately three times the combined flux from Tr176 and Triton. The half-power radius of the reference star's Gaussian was typically about 0.8 pixel. The reference star's PSF was fitted to the combined image of $\operatorname{Tr} 176$ and Triton, with the amplitude of the Gaussian and the coordinates of its center as free parameters. Note that here, as in the rest of the analysis, Triton and $\operatorname{Tr} 176$ are treated as a single source due to their proximity near and during the times of occultation. Data recorded before the occultation, when $\operatorname{Tr} 176$ and Triton were well separated, were used to estimate the separate signals of $\operatorname{Tr} 176$ and Triton and thus to calibrate the minimum flux from Tr176 at mid-occultation. However, the colors of Tr176, Triton, and the reference star are different, and differential extinction at the high airmass was important and not well calibrated owing to our inability to measure separate signals after occultation. We estimate the minimum flux from Tr176 at mid occultation to be $0.08 \pm 0.05$. Also, it should be noted that there was an interval of $\sim 10 \mathrm{~s}$, commencing at 10:10:30, when the reference star strayed from the $90 \times 90$ field due to guiding problems. During this interval, the PSF from the last fit to the reference star was used.

A light curve was constructed from the Chillagoe data cube with the PSF method, but Neptune was used as the standard. One problem with the Chillagoe data arose from variable windshake of the telescope. Individual images (Fig. 3) ranged in

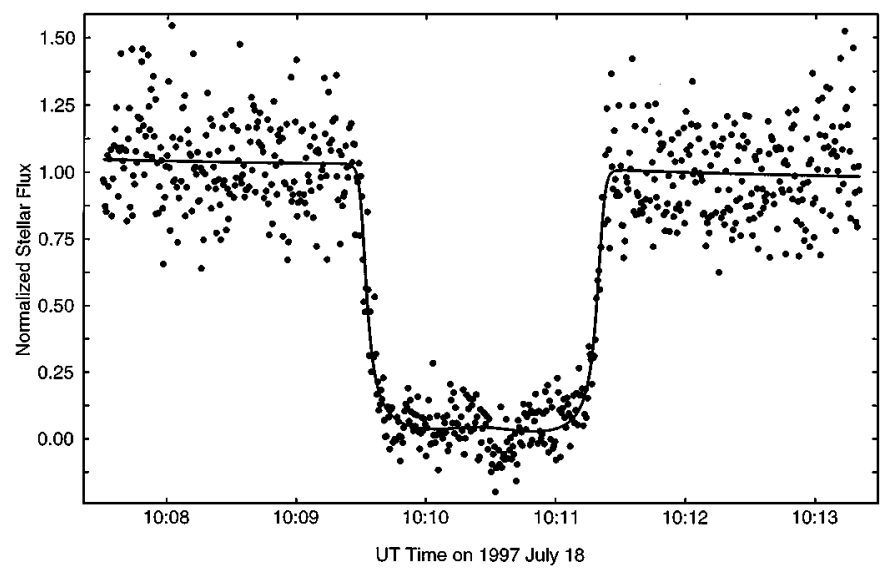

FIG . 4. Brownsville light curve: The normalized flux from $\operatorname{Tr} 176$ is plotted versus time after 1997 July $18,0^{\text {h }}$ UT. The solid line is a plot of a spherical, small-planet model (see text) fit to the entire light curve given in Table IX. The midtime was a free parameter (Table IX), which permitted the light curve to shift relative to the astrometric solution. The high scatter around the pre- and postoccultation signal levels is indicative of the signal-to-noise reported in Table IX. 
quality from small, compact, and easily distinguishable images of Neptune and the Triton-Tr176 blend to a smeared conglomeration of the two. To find an approximate position for Neptune's center we used the technique of marginal analysis (Elliot $e t$ al. 1989). In this method all the rows of the image frame are added to form a summed row, and the same procedure is applied to the columns. Then the image center in the summed row and the summed column is established. From this approximate center of Neptune we used the known offset of Triton to establish an approximate center of the Triton-Tr176 blend as starting values for the PSF fitting that would follow. Marginal analysis was applied to each frame, but at this stage marginal analysis failed or produced unusable results on the frames most affected by wind-shake. These frames were dropped from further analysis.

Once more accurate estimates of central pixel positions were obtained for each object on each frame and a least-squares procedure was used to simultaneously fit generalized Lorentzian PSFs to the image of Neptune and the blended images of Triton and $\operatorname{Tr} 176$ (which were treated as a single image). The image profile-described by Eq. (1) of Bosh et al. (1992)- has two parameters that describe the shape of the PSF: the diameter, $d$, of the image (defined as the full-width at half-maximum) and a power-law exponent, $p$ (termed the "shape index"). If $s_{0}$ is the peak signal and $r$ the radial distance from the center of the image, then the signal as a function of radial distance from the center of the image, $s(r)$ is given by

$$
s(r)=\frac{s_{0}}{1+(2 r / d)^{p}}
$$

In our modeling of the images of Neptune and the TritonTr176 blend, we fit for a common value of the shape index and image diameter. The row and column centers for both the blended image and that of Neptune were allowed to be free parameters, although the relative position of the blended image to that of Neptune was fixed at the average fitted value for 20 frames just after the occultation. Also used as free parameters were the background level, the peak signal level for Neptune, and the ratio of the Triton-Tr176 peak signal level to that of Neptune. Since this ratio is proportional to the signal of the star and Triton, corrected for variable seeing and extinction, we use it (versus time) for the occultation light curve.

This fit was applied to each of the remaining individual frames, although the least-squares fitting on some of them never converged. Some fits converged satisfactorily only after approximately 15 iterations, at which time the ratio of Triton-Tr176 to Neptune peak signal levels (with calculated error from the fit) were extracted for each frame and concatenated to form the light curve. The final light curve was then produced by discarding the points above a certain threshold in formal error in an attempt to eliminate those frames where wind-shake contaminated the Tr176-Triton image with smeared light from the wings of the Neptune image. After this, a light curve was compiled of points from these 1650 of the original 2000 frames acquired. A further

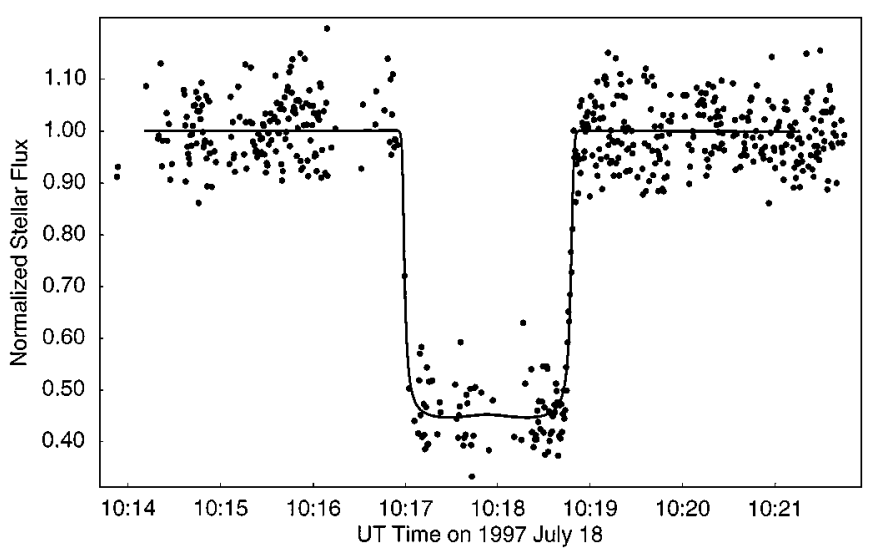

FIG. 5. Chillagoe light curve: The normalized ratio of the combined flux from Triton and $\operatorname{Tr} 176$ to that of Neptune is plotted versus time after July 18, $1997,0^{\mathrm{h}}$ UT. The solid line is a plot of a spherical, small-planet model (see text) fit to the entire light curve given in Table IX. The midtime was a free parameter (Table IX), which permitted the light curve to shift relative to the astrometric solution. The high scatter around the pre- and post-occultation signal levels is indicative of the signal-to-noise reported in table IX. The relative lack of points in the immersion part of the curve contributed to the high errors for the atmospheric parameters reported in Table XII.

830 points were trimmed from the pre- and post-occultation regions far from the event. The remaining points were further reduced by omitting the 285 with the largest formal errors in the ratio of the peak intensity of the blended image to that of Neptune. The resulting light curve is displayed in Fig. 5, where 535 of a possible 945 points within the time interval displayed were used in the subsequent analyses.

Construction of the Bundaberg, Ducabrook, and Lochington light curves will be described in a forthcoming publication on this event by Sicardy, Beisker, and their colleagues.

\section{POST-EVENT ASTROMETRY}

Light curves from all successful observation sites (Figs. 4-6) were then analyzed to produce an astrometric solution for the event. First the half-light times for immersion and emersion were established by a two-pass procedure. In this procedure, each light curve was fit with an isothermal, small-planet model (Elliot and Young 1992). The fitted parameters included only the full light level for Triton and Tr176, the background light level, the event mid-time, and the minimum distance between the site and the center of Triton's shadow, $\rho_{\min }$ (also referred to as the distance of closest approach). In these fits, the "energy ratio," $\lambda$ (the ratio of the gravitational potential energy of a molecule to $\mathrm{kT}$, Elliot and Young 1992), was fixed at 70, near the average value found by Olkin et al. (1997) for the highest quality data sets for the Tr148 occultation by Triton. Also, the half-light radius (in Triton's atmosphere) was fixed at $1450 \mathrm{~km}$. The half-light times were insensitive to the exact value of $\lambda$ and half-light radius used. For each light curve, the fitted event mid-times were then combined with the calculated shadow velocity and fitted closest 

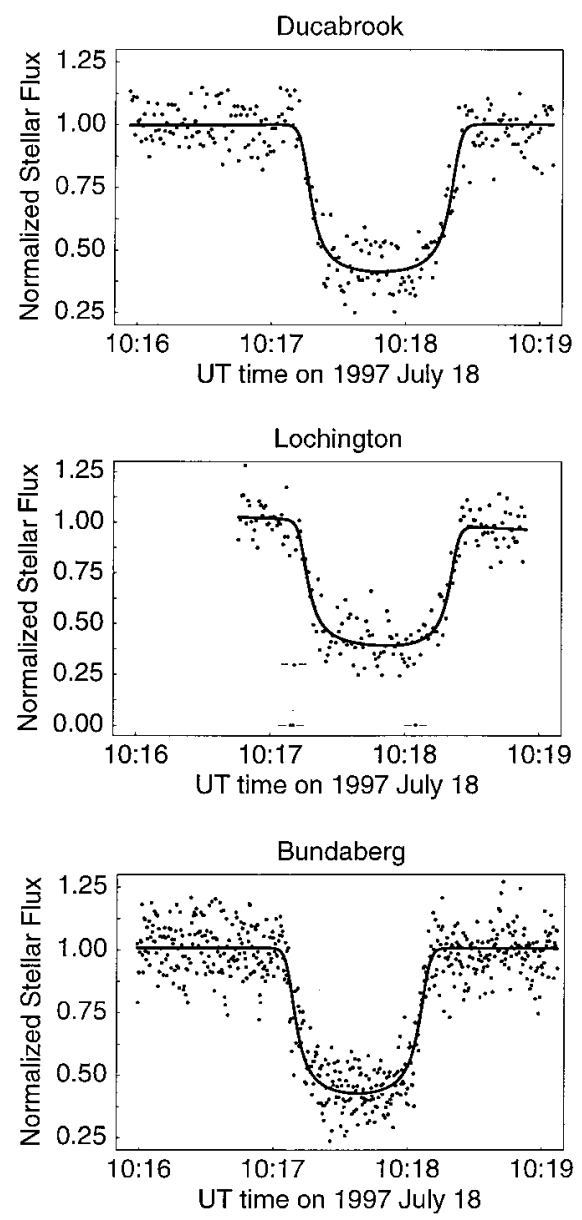

FIG . 6. IOTA light curves: The normalized flux of Triton and $\operatorname{Tr} 176$ is plotted versus time after 1997 July 18, $0^{\mathrm{h}}$ UT for the Bundaberg, Duckabrook, and Lochington light curves. The solid line is a plot of a spherical, small-planet model (see text) fit to the entire light curve given in Table IX. The midtime was a free parameter (Table IX), which permitted each light curve to shift relative to the astrometric solution. The high scatter around the pre- and post-occultation signal levels is indicative of the signal-to-noise reported in Table IX. The points noted were excluded from the Lochington fit.

approach distances to produce half-light times at immersion and emersion.

These half-light times were used in the astrometric reduction (described below), and then the light curves were fit again with the small-planet light curve model, but this time the closest approach distance $\left(\rho_{\min }\right)$ for each station was fixed at the value determined from the astrometric solution, but the half-light radius became a free parameter in the fit. Most of the resulting half-light times from the second set of fits differed less than $0.1 \mathrm{~s}$ from those in the first set. An exception was Bundaberg, where the differences between the two sets were 0.26 and $0.18 \mathrm{~s}$ for immersion and emersion, respectively. The half-light times from the second set of light-curve fits are given in Table $\mathrm{V}$, and these are the ones used in the subsequent steps of our analysis.

Our astrometric reduction was carried out in the $f g h$ system of Elliot et al. (1993). This is a geocentric coordinate system, with the $h$-axis pointing in the direction of the star, and the $f$ - and $g$-axes pointing in the direction of increasing right ascension and declination, respectively. In these calculations we used the celestial coordinates of $\operatorname{Tr} 176$ given by McDonald \& Elliot (1995) and JPL's DE405 ephemeris for Triton. Coordinates of the portable telescopes were determined by GPS receivers, and calculations are carried out in the J2000 coordinate system. Since the observer coordinates on Earth are referred to the Earth's instantaneous rotation pole (apparent coordinates) rather than the J2000 pole, one step of the calculation transformed the observer coordinates to the $\mathbf{J} 2000$ system. We prefer this approach to performing the reduction in apparent coordinates, since any small errors in the conversion of apparent coordinates to J2000 (i.e., precession, nutation, and the aberration of starlight) enter with a multiplicative factor of the Earth's radius, rather than the EarthTriton distance.

For the half-light times in Table $\mathrm{V}$ we calculated the topographic coordinates of the observer $\left[f_{\mathrm{r}}(t), g_{\mathrm{r}}(t)\right]$ and the coordinates of the center of Triton $\left[f_{\mathrm{p}^{\prime}}(t), g_{\mathrm{p}^{\prime}}(t)\right]$. We can relate these coordinates to the projected distance $\left[f_{\mathrm{pr}}(t), g_{\mathrm{pr}}(t)\right]$ between the center of Triton's shadow and the observer by allowing an (unknown) offset in Triton's ephemeris, $\left(f_{\mathrm{o}}, g_{\mathrm{o}}\right)$. These quantities are related by the equations

$$
\begin{aligned}
& f_{\mathrm{pr}}(t)=f_{\mathrm{r}}(t)-\left(f_{\mathrm{p}^{\prime}}(t)-f_{\mathrm{o}}\right) \\
& g_{\mathrm{pr}}(t)=g_{\mathrm{r}}(t)-\left(g_{\mathrm{p}^{\prime}}(t)-g_{\mathrm{o}}\right) .
\end{aligned}
$$

The coordinates $\left[f_{\mathrm{pr}}(t), g_{\mathrm{pr}}(t)\right]$ at the half-light times are given in Table $\mathrm{V}$, where each coordinate pair is either the immersion or the emersion location on the shadow plane for a single station. We can fit this set of coordinates to a circle, with the center coordinates $\left(f_{\mathrm{o}}, g_{\mathrm{o}}\right)$ and radius as free parameters. The resulting radius would be the half-light radius in the shadow plane (which is one scale-height smaller than the half-light radius in the atmosphere), and from the fitted values of $\left(f_{\mathrm{o}}, g_{\mathrm{o}}\right)$ we can derive the astrometric solution for the event (actual closest approach distances for each observing station).

We fit a circular figure to all 10 data points, which is labeled Fit No. 1 in Table VI. In this fit, $f_{\mathrm{o}}, g_{\mathrm{o}}$, and the radius of the shadow at half-light, $\rho_{\mathrm{h}}$, were free parameters. Carrying out this fit, we minimized the sum of squared residuals between each half-light point in the shadow plane and the model circle. Each residual was measured along the path traversed by each station within the shadow plane. In practice we find it easiest to calculate the residual, $\Delta s$, along the station path from the residual in radius, $\Delta r$, since the shadow path of each station is linear to a good approximation. If we denote the closest approach distance of a given station to the center of Triton's shadow by $\rho_{\min }$, then the residual $\Delta s$ is given by the equation

$$
\Delta s=\frac{\Delta r}{\sqrt{1-\left(\rho_{\min } / \rho_{\mathrm{h}}\right)^{2}}} .
$$

The quantities $\rho_{\min }$ and $\rho_{\mathrm{h}}$ in Eq. (4) can change with each iteration, so these are updated as the fit proceeds. 
TABLE VI

Astrometric Solutions ${ }^{a}$ from the Half-Light Timings

\begin{tabular}{|c|c|c|c|c|c|c|c|c|c|c|}
\hline $\mathrm{Fit}^{b}$ & Data selection ${ }^{c}$ & $f_{0},(\mathrm{~km})$ & $g_{0},(\mathrm{~km})$ & $\begin{array}{c}\text { Mean radius } \\
(\mathrm{km})\end{array}$ & $\begin{array}{l}\text { Semi-major } \\
\text { axis }(\mathrm{km})\end{array}$ & Ellipticity & $\begin{array}{l}\mathrm{PA}^{e} \\
(\mathrm{deg})\end{array}$ & $\mathrm{DoF}^{f}$ & $\begin{array}{c}\text { Reduced } \\
\chi^{2}\end{array}$ & $\begin{array}{c}\text { RMS error }{ }^{g} \\
(\mathrm{~km})\end{array}$ \\
\hline 1 & All & $-30917 \pm 18$ & $-4683 \pm 24$ & $1438 \pm 17$ & - & - & - & 7 & 15.08 & 31.6 \\
\hline 2 & Weights & $-30931 \pm 12$ & $-4688 \pm 15$ & $1439 \pm 10$ & - & - & - & 7 & 6.73 & 21.2 \\
\hline 3 & All & $-30929 \pm 5$ & $-4712 \pm 7$ & $1427 \pm 9$ & $1460 \pm 6$ & $0.046 \pm 0.007$ & -4.059 & 6 & 1.99 & 8.3 \\
\hline 4 & $\mathrm{PA}$ & $-30938 \pm 4$ & $-4693 \pm 6$ & $1437 \pm 11$ & $1467 \pm 4$ & $0.039 \pm 0.003$ & $23 \pm 6$ & 5 & 0.37 & 3.9 \\
\hline 5 & Weights & $-30935 \pm 5$ & $-4710 \pm 8$ & $1427 \pm 8$ & $1457 \pm 6$ & $0.042 \pm 0.008$ & -4.059 & 6 & 1.45 & 9.1 \\
\hline 6 & Weights PA & $-30940 \pm 2$ & $-4687 \pm 5$ & $1439 \pm 7$ & $1468 \pm 5$ & $0.040 \pm 0.003$ & $30 \pm 5$ & 5 & 0.24 & 3.4 \\
\hline
\end{tabular}

\footnotetext{
a These solutions are based on JPL's DE405 ephemeris for Triton and the celestial coordinates for Tr176 given by McDonald and Elliot (1995) for Tr176: $20 \mathrm{~h}$ $02 \mathrm{~m} 51.240 \mathrm{~s},-20^{\circ} 00^{\prime} 57.22^{\prime \prime}(\mathrm{J} 2000)$.

${ }^{b}$ Ellipticities and position angles are given for elliptical fits. All others are circular.

$c$ "PA" indicates that the position angle was fit freely instead of being fixed to Triton's pole position angle. "Weights" indicates that each data point was weighted inversely by the square of its formal error.

${ }^{d}$ This shadow-plane radius is one scale height smaller than the radius in Triton's atmosphere, due to refraction. For elliptical figures, this is the geometric mean of the two semi-axes.

${ }^{e}$ Position angle is defined as the angle of the semiminor axis from the north celestial pole, measured north through east.

${ }^{f}$ Degrees of freedom: the number of fitted data points minus the number of free parameters.

${ }^{g}$ The RMS error is defined as the square root of the weighted mean of the squared path residuals.
}

In the penultimate column of Table VI we display the "reduced chi-square," $\chi_{\text {red }}^{2}$, for this fit, which is defined as follows. If $\Delta s_{i}$ is the residual for the $i$ th data point, $v_{s i}$, the shadow velocity for the ith data point, $\sigma\left(t_{\mathrm{h} i}\right)$, the formal error for the $i$ th data point, $N_{\mathrm{d}}$, the number of fitted data points, and $N_{\mathrm{p}}$, the number of fitted parameters, then the reduced $\chi^{2}$ is given by

$$
\chi_{\mathrm{red}}^{2}=\frac{1}{N_{\mathrm{d}}-N_{\mathrm{p}}} \sum_{i=1}^{N_{\mathrm{d}}} \frac{\Delta s_{i}^{2}}{v_{s i}^{2} \sigma^{2}\left(t_{\mathrm{hi}}\right)} .
$$

The formal errors in the half-light times, $\sigma\left(t_{\mathrm{h} i}\right)$, are given in the last column of Table V.

Returning to our discussion of the reduced $\chi^{2}$, we note that it should be near 1.0 for a fit of an appropriate model to data with Gaussian noise of known standard deviation. Values that deviate far from 1.0 indicate that one of these assumptions is incorrect, with a probability that can be calculated or read from statistical tables (Bevington and Robinson 1992). Noting the high reduced $\chi^{2}$ in Table VI for Fit No. 1, we then tried fitting each point weighted inversely by the square of its formal error (Fit No. 2) with no better result. The residuals for all fits are given in Table VII.

Seeking improvement in the model fit, we proceeded to fit an elliptical model to the half-light points. In Fit No. 3 in Table VI we fit all the data with an elliptical figure that had the position angle of its semiminor axis fixed in the direction of Triton's rotation pole. The fit shows a significant ellipticity (apparent oblateness) of $0.046 \pm 0.007$ and a much lower $\chi^{2}$ than any of the circular fits that included the Brownsville data. In Fit No. 4 we allowed the position angle of the semiminor axis to be a free parameter, which allowed a significant rotation in the orientation of the apparent ellipse accompanied by a further reduction in the reduced $\chi^{2}$. Fits Nos. 5 and 6 are weighted versions of the previous two. Our conclusion from the elliptical fits is that we can achieve a plausible elliptical model for the figure of the halflight surface. However, the fitted orientation of the ellipse is not symmetric with respect to Triton's rotation axis, the expected line of symmetry. We adopt the weighted solutions (Fits Nos. 2 and 6) as our preferred circular and elliptical solutions. The mean half-light radii for these two are $1439 \pm 10$ and $1439 \pm$ $7 \mathrm{~km}$, respectively, and the implied closest approach distances for each solution are given in Table VIII and plotted in Fig. 7. There are only small differences between the closest approach distances implied by the two astrometric solutions, but to be definite we have adopted the closest approach distances for the circular solution for the subsequent steps in our analysis. We believe that the shadow center derived from the circular solution is likely to be a better indicator for the center of Triton's shadow

TABLE VII

Residuals (km) for the Astrometric Solutions

Fit Segment Brownsville Bundaberg Chillagoe Ducabrook Lochington

\begin{tabular}{llrrrrr}
\hline 1 & Immersion & 43.5 & -40.3 & 6.6 & -30.2 & -23.3 \\
1 & Emersion & -44.4 & 35.6 & -8.0 & 21.8 & 34.6 \\
2 & Immersion & 25.0 & -26.4 & -8.6 & -24.9 & -21.0 \\
2 & Emersion & -28.8 & 23.0 & 4.9 & 18.9 & 25.8 \\
3 & Immersion & 7.5 & -3.4 & -9.4 & -2.3 & 1.8 \\
3 & Emersion & 3.1 & 9.8 & -18.2 & 2.0 & 8.8 \\
4 & Immersion & -1.5 & -6.0 & 4.1 & -2.5 & 1.5 \\
4 & Emersion & 1.3 & 4.1 & -6.6 & -1.7 & 5.2 \\
5 & Immersion & 4.4 & -6.3 & -13.1 & -5.6 & -1.5 \\
5 & Emersion & 4.4 & 15.5 & -9.9 & 8.3 & 15.2 \\
6 & Immersion & -0.9 & -5.9 & 9.4 & -1.7 & 2.3 \\
6 & Emersion & 0.8 & 4.0 & -1.7 & -1.3 & 5.6 \\
\hline
\end{tabular}


TABLE VIII A dopted A strometric Solutions ${ }^{a}$ for Tr176

\begin{tabular}{lccc}
\hline \multicolumn{1}{c}{ Station } & $\begin{array}{c}\text { Closest approach } \\
\text { fit No. } 2(\mathrm{~km})\end{array}$ & $\begin{array}{c}\text { Closest approach } \\
\text { fit No. 6 }(\mathrm{km})\end{array}$ & $\begin{array}{c}\text { Shadow velocity } \\
\left(\mathrm{km} \mathrm{s}^{-1}\right)\end{array}$ \\
\hline Brownsville & $569.0 \pm 6.7$ & $564.4 \pm 10.2$ & 24.87 \\
Bundaberg & $1273.9 \pm 9.3$ & $1278.5 \pm 13.5$ & 24.90 \\
Chillagoe & $520.5 \pm 6.9$ & $525.0 \pm 11.1$ & 24.87 \\
Ducabrook & $1213.5 \pm 8.4$ & $1218.1 \pm 12.9$ & 24.88 \\
Lochington & $1217.6 \pm 8.3$ & $1222.2 \pm 12.2$ & 24.88 \\
\hline
\end{tabular}

\footnotetext{
${ }^{a}$ Based upon Fit Nos. 2 and 6 as listed in Table VI. The residuals are given in
} Table VII.

because this solution "averages" all half-light times. Due to the lack of more stations distributed across the shadow, we view the elliptical solution to be indicative of a non-circular atmospheric figure, but not a definitive solution, since the true figure could be more complex than a simple ellipse.

By comparison of the predicted and modeled closest approach distances and half-light times for the geocentric solution in Table III, we see that our final prediction was in error by $249 \mathrm{~km}$ (12 mas) in the direction perpendicular to the motion of Triton's shadow and $19 \mathrm{~s}$ (23 mas) in the direction parallel to the motion of Triton's shadow. These errors are somewhat larger than the formal error of the prediction (Table III).

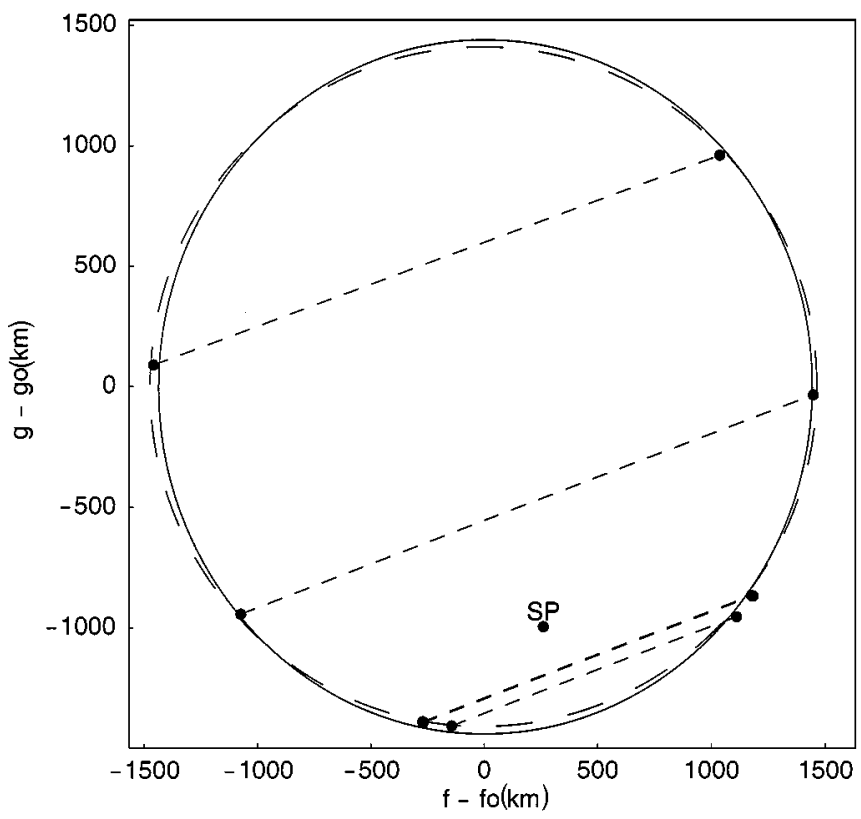

FIG . 7. Circular and elliptical atmospheric figures. The solid line represents the adopted circular solution and the dashed line the adopted elliptical solution (corresponding respectively to Fit Nos. 2 and 6 from Table VI). Scales given are kilometers north and east of the nominal Triton center from Fit No. 2. The chords from the five occultation stations are shown over the half-light disk of Triton. Note that the elliptical solution and the circular solution have different centers. The point (SP) in the southern hemisphere is Triton's south pole as seen from the occultation stations.

\section{LIGHT-CURVE ANALYSIS}

Work with past Triton occultation data showed that the smallplanet model of Elliot and Young (1992) provides a good description of the light curves (Elliot et al. 1997, 1998, Olkin et al. 1997), so we used this model to fit each light curve in three ways: (i) the entire light curve, (ii) immersion only, and (iii) emersion only. In each of these least-squares fits, the shadow velocity and the closest approach distance for each observing station to the center of Triton's shadow were fixed at the values from our adopted astrometric solution (Fit No. 2 of Table VI), but the following parameters were left free: full-scale signal level, background signal level, slope of the background signal level, half-light radius (within Triton's atmosphere), $r_{\mathrm{h}}$, and the parameter, $\lambda_{\mathrm{h}}$, where $\lambda_{\mathrm{h}}$ is the value of $\lambda$ at the half-light radius. The event midtime, $t_{\text {mid }}$, was a free parameter for the fits to entire light curves, but fixed at the mean of the half-light times (Table V) for the separate immersion and emersion fits.

If we define $H_{\mathrm{h}}$ as the equivalent-isothermal scale height at the half-light radius $\left(r_{\mathrm{h}}\right)$, in this model $H_{\mathrm{h}}=r_{\mathrm{h}} / \lambda_{\mathrm{h}}$ (Elliot and Young 1992). The data did not have sufficient signal-to-noise ratio to allow the thermal gradient to be a free parameter (as was possible for the Tr180 occultation, Elliot et al. 1998), so we assumed an isothermal atmosphere. Although we know that Triton's atmosphere is not isothermal over the altitude range probed by the occultation (Elliot et al. 2000), our results for atmospheric temperatures and pressures will be directly comparable with the equivalent-isothermal results from other occultations (Olkin et al. 1997).

The results of the model fits to the light curves are presented in Table IX-A, and plots of the model fits with the data all light curves are given in Figs. 4-6. In most cases, the results for the fit to the entire light curve are consistent with the results for immersion and emersion within expectations from their formal errors. However, the signal-to-noise ratios for these data sets are not high, in comparison with those for the Tr148 and Tr180 occultations, which involved brighter stars and larger telescopes. In both of these other occultations, the atmosphere exhibited the same temperature and pressure at all points probed to the noise limit of the light curves within these data sets (Olkin et al. 1997, Elliot et al. 1998, 2000). The sub-occultation locations on Triton that were probed by this occultation are given in Table X. Using values for parameters given in Table XI, we have used the results of the model fits in Table IX-A to derive the number densities, pressures, and temperatures for an equivalent-isothermal atmosphere (Table XII-A) for the immersion and emersion fits. The symbols $p_{1400}$ and $n_{1400}$ denotes the pressure and number density at a radius of $1400 \mathrm{~km}$ (altitude of $48 \mathrm{~km}$ ), which is the approximate level for which the pressure derived from the model-fit parameters is the most accurate.

Clearly some of the results (e.g., emersion for Bundaberg and immersion for Chillagoe and Lochington) have very large errors, and these results are highly questionable at best. The reason for these large error bars is that signal-to-noise ratios of 
TABLE IX-A

Model Parameters from Circular Fits of the Light Curves

\begin{tabular}{|c|c|c|c|c|c|c|}
\hline Parameter & Light curve & Brownsville ${ }^{e}$ & Bundaberg $^{e}$ & Chillagoe $^{f}$ & Ducabrook $^{e}$ & Lochington $^{e}$ \\
\hline \multirow[t]{3}{*}{ Full signal } & Full & $1.01 \pm 0.01$ & $1.01 \pm 0.00$ & $1.00 \pm 0.01$ & $0.99 \pm 0.01$ & $0.99 \pm 0.01$ \\
\hline & Immersion & $0.99 \pm 0.04$ & $1.01 \pm 0.01$ & $1.03 \pm 0.02$ & $1.02 \pm 0.01$ & $0.85 \pm 0.09$ \\
\hline & Emersion & $1.00 \pm 0.05$ & $0.96 \pm 0.03$ & $1.01 \pm 0.01$ & $0.94 \pm 0.05$ & $1.03 \pm 0.09$ \\
\hline \multirow[t]{3}{*}{ Signal slope } & Full & $-1.84 \pm 0.55 \times 10^{-4}$ & $-6.42 \pm 3.58 \times 10^{-5}$ & $5.55 \pm 3.82 \times 10^{-5}$ & $1.22 \pm 5.24 \times 10^{-5}$ & $-6.59 \pm 2.71 \times 10^{-4}$ \\
\hline & Immersion & $-4.06 \pm 2.82 \times 10^{-4}$ & $-3.78 \pm 2.09 \times 10^{-4}$ & $3.12 \pm 1.80 \times 10^{-6}$ & $1.84 \pm 0.96 \times 10^{-4}$ & $-4.22 \pm 2.54 \times 10^{-3}$ \\
\hline & Emersion & $-1.81 \pm 6.02 \times 10^{-4}$ & $1.51 \pm 1.14 \times 10^{-3}$ & $-6.64 \pm 8.08 \times 10^{-4}$ & $1.08 \pm 0.89 \times 10^{-3}$ & $-1.74 \pm 2.41 \times 10^{-3}$ \\
\hline \multirow[t]{3}{*}{ Background } & Full & $-0.06 \pm 0.02$ & $0.34 \pm 0.01$ & $0.42 \pm 0.03$ & $0.33 \pm 0.00$ & $0.31 \pm 0.01$ \\
\hline & Immersion & $-0.07 \pm 0.03$ & $0.30 \pm 0.01$ & $0.42 \pm 0.03$ & $0.34 \pm 0.01$ & $0.34 \pm 0.01$ \\
\hline & Emersion & $0.01 \pm 0.04$ & $0.38 \pm 0.01$ & $0.43 \pm 0.03$ & $0.31 \pm 0.02$ & $0.28 \pm 0.02$ \\
\hline \multirow[t]{3}{*}{$r_{\mathrm{h}}(\mathrm{km})$} & Full & $1459.6 \pm 6.3$ & $1458.4 \pm 3.8$ & $1453.3 \pm 6.1$ & $1455.3 \pm 5.5$ & $1462.1 \pm 5.6$ \\
\hline & Immersion & $1462.1 \pm 9.7$ & $1455.5 \pm 6.9$ & $1448.5 \pm 13.1$ & $1460.0 \pm 6.9$ & $1466.5 \pm 7.9$ \\
\hline & Emersion & $1459.2 \pm 7.9$ & $1454.0 \pm 4.2$ & $1453.4 \pm 4.9$ & $1447.2 \pm 9.2$ & $1454.2 \pm 7.6$ \\
\hline \multirow[t]{3}{*}{$\lambda_{\mathrm{h}}$} & Full & $52.4 \pm 8.1$ & $69.9 \pm 9.6$ & $108.5 \pm 22.1$ & $60.4 \pm 10.3$ & $66.3 \pm 12.3$ \\
\hline & Immersion & $48.9 \pm 11.2$ & $53.5 \pm 10.7$ & $124.3 \pm 51.3$ & $69.8 \pm 17.0$ & $112.1 \pm 56.2$ \\
\hline & Emersion & $71.2 \pm 18.1$ & $85.0 \pm 18.9$ & $93.2 \pm 18.6$ & $56.0 \pm 16.7$ & $56.6 \pm 17.1$ \\
\hline$t_{\text {mid }}{ }^{a}$ & Full & $10: 10: 25.26 \pm 0.27$ & $10: 17: 37.71 \pm 0.26$ & $10: 17: 53.24 \pm 0.27$ & $10: 17: 48.83 \pm 0.34$ & $10: 17: 48.39 \pm 0.37$ \\
\hline$t_{\text {mid }}{ }^{a}$ & Im and em & 10:10:25.38 & $10: 17: 37.73$ & 10:17:53.17 & $10: 17: 48.83$ & 10:17:48.76 \\
\hline$\rho_{\min }(\mathrm{km})$ & All & 577.4 & 1266.5 & 514.1 & 1206.4 & 1210.5 \\
\hline$v\left(\mathrm{~km} \mathrm{~s}^{-1}\right)$ & All & 24.87 & 24.90 & 24.87 & 24.88 & 24.88 \\
\hline $\operatorname{DoF}^{b}$ & Full & 695 & 1887 & 528 & 505 & 175 \\
\hline Residuals $^{c}$ & Full & 1.91 & 3.95 & 1.39 & 1.84 & 1.14 \\
\hline $\mathrm{SNR}^{d}$ & Full & 24.2 & 34.1 & 24.7 & 17.6 & 13.2 \\
\hline
\end{tabular}

\footnotetext{
${ }^{a}$ Time after July 18, 1997, 0 h UTC. Mid-time was freely fit to the full light-curve data. Immersion and emersion fits had a fixed mid-time. Fixed mid-times were taken from previous fits to light curves needed for the astrometric analysis.

${ }^{b}$ Degrees of freedom.

${ }^{c}$ Square root of the sum of the squared residuals. (Residuals normalized to full signal level.)

${ }^{d}$ Ratio of signal for a $20-\mathrm{km}$ portion of the shadow path to noise as calculated from the given fit residuals.

${ }^{e}$ Light-curve signal parameters were normalized to full signal level prior to fitting. Differences between full signal levels and 1.00 are the result of fitting light curves to models after this initial normalization.

${ }^{f}$ Light-curve parameters were normalized to full signal level after fitting. Original full signal level and normalization factor (the ratio of Tr176 + Triton flux to that of Neptune determined by earlier fits) was $0.0317 \pm 0.0001$.
}

these data are barely sufficient for the least-squares fitting that we have carried out, and-in the case of Chillagoe-the problem is exacerbated by a loss of data during the main immersion drop of the light curve caused by wind-shake. Numerical experiments with synthetic light curves and computer-generated random noise produce similar results for the noise levels encountered in these data.

The pressure at $1400 \mathrm{~km}$ (48 km altitude) is the most sensitive indicator for comparison with other pressures derived from occultation data to determine whether changes in atmospheric pressure have occurred (Olkin et al. 1997). The weighted average (equivalent-isothermal) pressure at $1400 \mathrm{~km}$ is $2.23 \pm 0.28 \mu \mathrm{bar}$ for the circular model. Since the pressure errors are somewhat large and the differences between stations are not significant, we use this weighted average for comparison with the results from other occultations.

Triton's atmospheric figure is distorted from a sphere, so we also derive the pressure using an ellipse to describe the first-order deviation from a circular shape. For this analysis, we assume that the half-light surface in Triton's atmosphere is an isobar (to first order), and we use this "half-light isobar" as a reference for inferring the pressure at other levels. The results from this analysis based on an elliptical atmospheric figure can be most directly compared with the results based on circular figures if we calculate pressures for an elliptical isobar that has a mean radius of $1400 \mathrm{~km}$. We shall refer to this as the $1400-\mathrm{km}$ ellipse.

To determine pressures on this isobar, analysis proceeded as described for a circular solution except that the isothermal lightcurve models were generated using an elliptical figure for the refractivity contours and calculating fluxes from all perpendicular limb points on this contour as outlined by Elliot et al. (1997) for modeling the central flash data for the Tr148 occultation. The individual elliptical model fits for the various stations are listed in Table IX-B.

For extrapolation from the half-light level, each occultation event was treated separately (immersions and emersions) for the five stations. For each event the local half-light radius was calculated from the equatorial radius, ellipticity, and position angle of our adopted elliptical model (Fit No. 6 in Table VI). This local half-light radius was then combined with the fitted energy 
TABLE IX-B

Model Parameters from Elliptical Fits of the Light C urves

\begin{tabular}{|c|c|c|c|c|c|c|}
\hline Parameter & Light curve & Brownsville $^{e}$ & Bundaberg $^{e}$ & Chillagoe $^{f}$ & Ducabrook $^{e}$ & Lochington $^{e}$ \\
\hline Full signal & $\begin{array}{l}\text { Full } \\
\text { Immersion } \\
\text { Emersion }\end{array}$ & $\begin{array}{l}1.01 \pm 1.01 \\
0.99 \pm 0.01 \\
0.99 \pm 0.06\end{array}$ & $\begin{array}{l}1.01 \pm 0.02 \\
1.01 \pm 0.01 \\
0.96 \pm 0.24\end{array}$ & $\begin{array}{l}1.00 \pm 0.01 \\
1.00 \pm 0.02 \\
1.00 \pm 0.01\end{array}$ & $\begin{array}{l}0.33 \pm 0.02 \\
1.02 \pm 0.01 \\
0.94 \pm 0.01\end{array}$ & $\begin{array}{l}0.99 \pm 0.01 \\
0.86 \pm 0.09 \\
1.03 \pm 0.09\end{array}$ \\
\hline Signal slope & $\begin{array}{l}\text { Full } \\
\text { Immersion } \\
\text { Emersion }\end{array}$ & $\begin{array}{l}-1.52 \pm 0.59 \times 10^{-4} \\
-4.10 \pm 2.82 \times 10^{-4} \\
-1.85 \pm 0.60 \times 10^{-4}\end{array}$ & $\begin{array}{r}-2.05 \pm 1.02 \times 10^{-5} \\
-1.56 \pm 6.59 \times 10^{-5} \\
1.50 \pm 1.07 \times 10^{-3}\end{array}$ & $\begin{array}{r}-2.69 \pm 2.03 \times 10^{-5} \\
2.26 \pm 1.08 \times 10^{-4} \\
-2.27 \pm 4.34 \times 10^{-4}\end{array}$ & $\begin{array}{l}1.06 \pm 3.56 \times 10^{-5} \\
1.28 \pm 0.02 \times 10^{-4} \\
9.49 \pm 3.26 \times 10^{-4}\end{array}$ & $\begin{array}{l}-2.84 \pm 1.42 \times 10^{-4} \\
-2.84 \pm 0.08 \times 10^{-3} \\
-1.15 \pm 1.69 \times 10^{-3}\end{array}$ \\
\hline Background & $\begin{array}{l}\text { Full } \\
\text { Immersion } \\
\text { Emersion }\end{array}$ & $\begin{array}{r}-0.06 \pm 0.02 \\
-0.06 \pm 0.04 \\
0.01 \pm 0.03\end{array}$ & $\begin{array}{l}0.34 \pm 0.02 \\
0.32 \pm 0.03 \\
0.37 \pm 0.02\end{array}$ & $\begin{array}{l}0.42 \pm 0.01 \\
0.43 \pm 0.02 \\
0.33 \pm 0.01\end{array}$ & $\begin{array}{l}1.00 \pm 0.01 \\
0.35 \pm 0.03 \\
0.31 \pm 0.04\end{array}$ & $\begin{array}{l}0.32 \pm 0.01 \\
0.49 \pm 0.03 \\
0.28 \pm 0.05\end{array}$ \\
\hline$r_{\mathrm{h}}(\mathrm{km})$ & $\begin{array}{l}\text { Full } \\
\text { Immersion } \\
\text { Emersion }\end{array}$ & $\begin{array}{l}1491.7 \pm 6.5 \\
1494.6 \pm 9.9 \\
1491.5 \pm 8.1\end{array}$ & $\begin{array}{l}1489.1 \pm 3.9 \\
1488.3 \pm 6.9 \\
1484.9 \pm 4.3\end{array}$ & $\begin{array}{l}1490.7 \pm 4.6 \\
1484.2 \pm 13.2 \\
1489.3 \pm 5.1\end{array}$ & $\begin{array}{l}1487.2 \pm 5.5 \\
1492.4 \pm 7.1 \\
1477.7 \pm 9.6\end{array}$ & $\begin{array}{l}1494.1 \pm 5.8 \\
1498.8 \pm 8.1 \\
1485.2 \pm 7.9\end{array}$ \\
\hline$\lambda_{\mathrm{h}}$ & $\begin{array}{l}\text { Full } \\
\text { Immersion } \\
\text { Emersion }\end{array}$ & $\begin{array}{l}53.1 \pm 8.2 \\
52.3 \pm 12.0 \\
67.9 \pm 17.3\end{array}$ & $\begin{array}{c}69.1 \pm 9.5 \\
58.3 \pm 11.4 \\
110.6 \pm 27.5\end{array}$ & $\begin{array}{r}102.3 \pm 20.4 \\
176.3 \pm 69.2 \\
89.7 \pm 17.9\end{array}$ & $\begin{array}{l}61.2 \pm 10.5 \\
75.8 \pm 18.3 \\
50.1 \pm 15.0\end{array}$ & $\begin{array}{r}65.4 \pm 12.3 \\
121.6 \pm 40.7 \\
51.6 \pm 15.8\end{array}$ \\
\hline $\begin{array}{l}t_{\text {mid }}{ }^{a} \\
\lambda_{\min }(\mathrm{km}) \\
v\left(\mathrm{~km} \mathrm{~s}^{-1}\right)\end{array}$ & $\begin{array}{l}\text { All } \\
\text { All } \\
\text { All }\end{array}$ & $\begin{array}{c}10: 10: 25.38 \\
564.4 \\
24.87\end{array}$ & $\begin{array}{c}10: 17: 37.73 \\
1278.5 \\
24.90\end{array}$ & $\begin{array}{c}10: 17: 53.17 \\
525.0 \\
24.87\end{array}$ & $\begin{array}{c}10: 17: 48.83 \\
1218.1 \\
24.88\end{array}$ & $\begin{array}{c}10: 17: 48.76 \\
1222.2 \\
24.88\end{array}$ \\
\hline $\begin{array}{l}\operatorname{DoF}^{b} \\
\text { Residuals }^{c} \\
\text { SNR }^{d}\end{array}$ & $\begin{array}{l}\text { Full } \\
\text { Full } \\
\text { Full }\end{array}$ & $\begin{array}{l}696 \\
1.91 \\
24.2\end{array}$ & $\begin{array}{l}1888 \\
3.95 \\
34.1\end{array}$ & $\begin{array}{l}529 \\
1.39 \\
24.7\end{array}$ & $\begin{array}{l}506 \\
1.84 \\
17.6\end{array}$ & $\begin{array}{l}171 \\
1.15 \\
13.0\end{array}$ \\
\hline
\end{tabular}

${ }^{a}$ Time after July 18, 1997, 0 h UTC. Fixed mid-times were taken from previous fits to light curves used in the astrometric analysis.

${ }^{b}$ Degrees of freedom.

${ }^{c}$ Square root of the sum of the squared residuals. (Residuals normalized to full signal level.)

${ }^{d}$ Ratio of signal for a $20-\mathrm{km}$ portion of the shadow path to noise as calculated from the given fit residuals.

${ }^{e}$ Light-curve signal parameters were normalized to full signal level prior to fitting. Differences between full signal levels and 1.00 are the result of fitting light curves to models after this initial normalization.

${ }^{f}$ Light-curve parameters were normalized to full signal level after fitting. Original full signal level and normalization factor (the ratio of Tr176 + Triton flux to that of Neptune determined by earlier fits) was $0.0330 \pm 0.0005$.

ratio $\left(\lambda_{h}\right)$ to determine the local (equivalent isothermal) pressure scale height. This scale height was then used to extrapolate the pressure to the 1400-km ellipse (along the pressure gradient), which is $p_{1400}$ for the elliptical model. These pressures are given in Table XII-B, and their weighted mean is $2.45 \pm 0.32 \mu$ bar.

\section{COMPARISON AND DISCUSSION}

In this section we compare our result for Triton's non-spherical atmospheric figure and its atmospheric pressure with results

TABLE X

Triton Longitude and Latitude Probed at Half Light by Tr176

\begin{tabular}{lccccc}
\hline & \multicolumn{2}{c}{ Immersion } & & \multicolumn{2}{c}{ Emersion } \\
\cline { 2 - 3 } \cline { 5 - 6 } \multicolumn{1}{c}{ Site } & $\begin{array}{c}\text { Longitude } \\
\text { (deg.) }\end{array}$ & $\begin{array}{c}\text { Latitude } \\
\text { (deg.) }\end{array}$ & & $\begin{array}{c}\text { Longitude } \\
\text { (deg.) }\end{array}$ & $\begin{array}{c}\text { Latitude } \\
\text { (deg.) }\end{array}$ \\
\hline Brownsville & 9.1 & +4.6 & & 226.9 & +24.4 \\
Bundaberg & 89.5 & -39.4 & & 156.1 & -27.9 \\
Chillagoe & 44.4 & -23.0 & & 190.5 & -3.5 \\
Ducabrook & 83.1 & -38.4 & & 160.3 & -25.7 \\
Lochington & 82.8 & -38.3 & & 160.2 & -25.7 \\
\hline
\end{tabular}

from other stellar occultations by Triton. We also discuss our interpretations of these findings.

\section{Atmospheric Figure}

The parameters describing our adopted elliptical solution (Fit No. 6 of Table VI) have been summarized in Table XIII. Also entered in this table are the parameters for elliptical solutions from the $\operatorname{Tr} 148$ occultation, both from fitting an elliptical figure to the shadow-plane coordinates of the half-light times (Olkin et al. 1997) and from modeling the central flash (Elliot et al. 1997). In their analysis, Olkin et al. (1997) concluded that the half-light elliptical solution was not significant because of the relatively

\section{TABLE XI}

\section{Parameters for $C$ onversion of $F$ it $R$ esults}

\begin{tabular}{lcl}
\hline \multicolumn{1}{c}{ Parameter } & Value & Reference \\
\hline Geocentric distance to Triton, AU & 29.169 & JPL DE405, NEP016 \\
Triton mass, kg & $2.139810^{22}$ & (Anderson et al. 1992) \\
Molecular weight of $\mathrm{N}_{2}$, amu & 28.01 & \\
$\mathrm{~N}_{2}$ refractivity, $v_{\mathrm{STP}}$ at $0.7 \mu \mathrm{m}$ & $2.9810^{-4}$ & (Peck and Khanna 1966)
\end{tabular}


TABLE XII-A

E quivalent-I sothermal Atmospheric Parameters (C ircular Solutions)

\begin{tabular}{llccccc}
\hline \multicolumn{1}{c}{ Parameter } & Fit & Brownsville & Bundaberg & Chillagoe & Ducabrook & Lochington \\
\hline$r_{\mathrm{h}}(\mathrm{km})$ & & & & & & \\
& Im & $1462.1 \pm 9.7$ & $1455.5 \pm 6.9$ & $1448.5 \pm 13.1$ & $1460.0 \pm 6.9$ & $1466.5 \pm 7.9$ \\
$\lambda_{\mathrm{h}}$ & Em & $1459.2 \pm 7.9$ & $1454.0 \pm 4.2$ & $1453.4 \pm 4.9$ & $1447.2 \pm 9.2$ & $1454.2 \pm 7.6$ \\
& Im & $48.9 \pm 11.2$ & $53.5 \pm 10.7$ & $124.3 \pm 51.3$ & $69.8 \pm 17.0$ & $112.1 \pm 56.2$ \\
$H_{\mathrm{h}}(\mathrm{km})$ & Em & $71.2 \pm 18.1$ & $85.0 \pm 18.9$ & $93.2 \pm 18.6$ & $56.0 \pm 16.7$ & $56.6 \pm 17.1$ \\
& Im & $29.9 \pm 6.8$ & $27.2 \pm 5.4$ & $8.8 \pm 9.8$ & $20.9 \pm 5.1$ & $13.0 \pm 6.5$ \\
$T_{\text {iso }}(\mathrm{K})$ & Em & $20.5 \pm 5.2$ & $17.0 \pm 3.0$ & $15.6 \pm 3.1$ & $25.8 \pm 7.6$ & $25.7 \pm 7.7$ \\
& Im & $67.2 \pm 15.3$ & $61.7 \pm 12.4$ & $20.2 \pm 22.1$ & $47.2 \pm 11.5$ & $29.1 \pm 14.5$ \\
$n_{1400}\left(10^{11} \mathrm{~cm}^{-3}\right)^{a}$ & Em & $46.3 \pm 11.8$ & $37.3 \pm 8.7$ & $35.5 \pm 7.1$ & $59.3 \pm 17.6$ & $58.4 \pm 17.7$ \\
& Im & $3.22 \pm 1.21$ & $2.65 \pm 0.89$ & $17.05 \pm 60.24$ & $4.24 \pm 2.31$ & $21.82 \pm 44.04$ \\
$p_{1400}\left(\mu\right.$ bar $^{b}$ & Em & $4.19 \pm 2.24$ & $9.73 \pm 8.56$ & $4.77 \pm 2.49$ & $1.95 \pm 0.80$ & $2.61 \pm 1.06$ \\
& Im & $2.99 \pm 1.05$ & $2.25 \pm 0.55$ & $4.76 \pm 11.72$ & $2.76 \pm 1.06$ & $8.76 \pm 13.55$ \\
& Em & $2.67 \pm 1.10$ & $3.67 \pm 2.43$ & $2.33 \pm 0.90$ & $1.60 \pm 0.61$ & $2.10 \pm 0.62$ \\
\hline
\end{tabular}

${ }^{a}$ Number density (for $\mathrm{N}_{2}$ ) at $1400 \mathrm{~km}$.

${ }^{b}$ Pressure (for $\mathrm{N}_{2}$ ) at $1400 \mathrm{~km}$.

large formal errors in the fitted parameters. We agree with this conclusion but it may be indicating some non-spherical atmospheric structure, so we have shown the solution in Table XIII for reference.

In comparing the three ellipses presented in Table XIII, we see that none of the solutions agree with each other. Furthermore, only the central-flash solution (for Tr148) has a semiminor axis that is consistent with the direction of Triton's pole at the time-a line of symmetry that might be preferred for global wind patterns. The half-light solutions refer to a radius of about $1450 \mathrm{~km}$ in Triton's atmosphere (which corresponds to an altitude of about $100 \mathrm{~km}$ ), while central-flash modeling is sensitive to the shape of the atmosphere several scale heights lower, at an altitude of about $20 \mathrm{~km}$. Hence, we must allow for the possibility that-depending on the dynamical state of Triton's atmosphere-isobaric surfaces could have different figures at different altitudes. Note that for the purposes of this discussion we are assuming that the half-light surface is an isobaric surface, which need not be the case but is likely a good approximation.

We conclude that at the times of the $\operatorname{Tr} 148$ and $\operatorname{Tr} 176$ occultations, Triton's atmosphere has exhibited a significantly nonspherical figure. However, the size and orientation of the ellipses at those two times are not consistent with each other, and the ellipticities differ by a factor of 2 . Here we also note that the observed ellipticities are lower limits on the oblateness of Triton's three-dimensional figure, since the observed ellipticities represent the three-dimensional oblateness projected onto the shadow plane.

TABLE XII-B

E quivalent-I sothermal Atmospheric Parameters (E Iliptical Solutions)

\begin{tabular}{llccccc}
\hline \multicolumn{1}{c}{ Parameter } & Fit & Brownsville & Bundaberg & Chillagoe & Ducabrook & Lochington \\
\hline$r_{\mathrm{h}}($ local; $\mathrm{km})$ & Im & 1482.1 & 1438.5 & 1430.5 & 1438.9 & 1445.1 \\
& Em & 1436.8 & 1482.8 & 1474.9 & 1477.0 & 1484.6 \\
$\lambda_{\mathrm{h}}$ & Im & $52.3 \pm 12.0$ & $58.3 \pm 11.4$ & $176.3 \pm 189.2$ & $75.8 \pm 18.3$ & $121.6 \pm 60.7$ \\
& Em & $67.9 \pm 17.2$ & $110.6 \pm 27.5$ & $89.7 \pm 17.9$ & $50.1 \pm 15.0$ & $51.6 \pm 15.8$ \\
$H_{\mathrm{h}}(\mathrm{km})$ & $\mathrm{Im}$ & $28.3 \pm 6.5$ & $24.7 \pm 4.8$ & $8.1 \pm 8.7$ & $18.9 \pm 4.6$ & $11.9 \pm 5.9$ \\
& Em & $21.1 \pm 5.4$ & $13.4 \pm 3.3$ & $16.4 \pm 3.3$ & $29.5 \pm 8.8$ & $28.8 \pm 8.8$ \\
$T_{\text {iso }}(\mathrm{K})$ & Im & $62.0 \pm 14.2$ & $57.4 \pm 11.3$ & $19.1 \pm 20.2$ & $44.1 \pm 10.6$ & $27.4 \pm 13.6$ \\
& Em & $49.3 \pm 12.5$ & $29.3 \pm 7.3$ & $36.4 \pm 7.3$ & $65.0 \pm 19.6$ & $62.8 \pm 19.3$ \\
$n_{1400}\left(10^{11} \mathrm{~cm}^{-3}\right)^{a}$ & Im & $3.72 \pm 1.56$ & $3.11 \pm 1.19$ & $47.0 \pm 48.3$ & $5.37 \pm 3.38$ & $34.7 \pm 79.7$ \\
$p_{1400}\left(\mu \mathrm{bar}^{b}\right.$ & Em & $4.28 \pm 2.22$ & $8.18 \pm 6.55$ & $6.50 \pm 3.58$ & $1.98 \pm 0.71$ & $2.63 \pm 0.96$ \\
& Im & $3.19 \pm 1.17$ & $2.47 \pm 0.67$ & $12.37 \pm 13.25$ & $3.27 \pm 1.49$ & $13.10 \pm 23.90$ \\
& Em & $2.91 \pm 1.15$ & $3.31 \pm 1.93$ & $3.26 \pm 1.32$ & $1.78 \pm 0.63$ & $2.28 \pm 0.64$ \\
\hline
\end{tabular}

${ }^{a}$ Number density (for $\mathrm{N}_{2}$ ) on the ellipse with mean radius $1400 \mathrm{~km}$.

${ }^{b}$ Pressure (for $\mathrm{N}_{2}$ ) on the ellipse with mean radius $1400 \mathrm{~km}$. 
TABLE XIII

Comparison of Elliptical Models

\begin{tabular}{lcccrr}
\hline Elliptical model & Event date & Semimajor axis $^{a}(\mathrm{~km})$ & Ellipticity & PA of semi-minor axis & PA of Triton pole $\left({ }^{\circ}\right)$ \\
\hline Tr148, central flash & $1995-08-14$ & $1432 \pm 2$ & $0.018 \pm 0.003$ & $3 \pm 5$ & 3.2 \\
Tr148, half-light & $1995-08-14$ & $(1475 \pm 6)^{b}$ & $(0.029 \pm 0.016)$ & $(-20 \pm 10)$ & (Elliot et al. 1997) \\
Tr176, half-light & $1997-07-18$ & $1468 \pm 5$ & $0.040 \pm 0.003$ & $30 \pm 5$ & $($ Olkin et al. 1997) \\
\hline
\end{tabular}

${ }^{a}$ Half-light level in the shadow plane.

${ }^{b}$ Values in parentheses have low statistical significance.

The oblateness (ellipticity) $e$ of a planetary atmosphere is related to the gravitational moment $J_{2}$ and the rotational parameter $q$ by

$$
e=\frac{3}{2} J_{2}+\frac{q}{2},
$$

where $q=v^{2} / a g$. Here $v$ is the equatorial rotation speed, $a$ is the mean radius, and $g$ is the atmospheric gravity. The lack of correlation between the orientation of the minor axis of the atmospheric figure and the rotation axis of Triton indicates that the contribution of $J_{2}$ to $e$ must be negligible. This empirical conclusion agrees with our calculations using the model of Zharkov et al. (1985) that the rotation of Triton and tidal forces from Neptune would produce an ellipticity of only $\sim 0.001$. Setting $J_{2}=0$ in Eq. (6), we find that $v=280 \mathrm{~m} \mathrm{~s}^{-1}$ for $e=0.040$ (Fit No. 6 in Table VI), compared with a sound speed of $140 \mathrm{~m} \mathrm{~s}^{-1}$ ( $v$ would be much lower if the atmosphere were rotating with the solid body). It seems highly unlikely that this is a permanent state of affairs in Triton's atmosphere, for the equator-to-pole variation in the pressure at a fixed altitude would be comparable to the pressure itself!

A similar unexpectedly large ellipticity (0.016) was observed for Titan at the time of the $28 \mathrm{Sgr}$ occultation (Hubbard et al. 1993). Although the multi-chord observations of the central flash were not consistent with a simple elliptical model, Hubbard et al. (1993) constructed a differentially rotating model that satisfied the multiple central-flash observations and required maximum wind speeds about half that for the best fitting oblate model. In their analysis of the shape of the central flash for the $\operatorname{Tr} 148$ occultation, Elliot et al. (1997) presented arguments regarding why the observed ellipticity could not be due to a distortion of Triton's gravity field, Triton's atmospheric extinction, or variable refraction due to a partial segregation of Triton's atmospheric species. They concluded that a global elliptical model implied wind speeds in excess of the sonic speed and proposed that winds restricted to certain latitude bands (essentially a differentially rotating model as discussed above for Titan) could explain the data without requiring such high wind speeds. Unfortunately, only a single light curve probed the central flash, so a more detailed model for the winds on Triton could not be constructed as Hubbard et al. (1993) were able to do for Titan.

Several lines of evidence from the Voyager encounter established the existence of winds on Triton: (i) the surface streaks
(Hansen et al. 1990), (ii) the observed motion of a plume top (Soderblom et al. 1990), and (iii) the possible motion of a cloud (which has an alternative interpretation as different visible portions of a larger stationary cloud, Hansen et al. 1990). Each of these indicators gives a wind direction, while wind speeds derived from the latter two are 15 and $13 \mathrm{~m} \mathrm{~s}^{-1}$, respectively (Hansen et al. 1990, Soderblom et al. 1990). Atmospheric distortion is yet another indicator of winds, but, as discussed above, speeds in excess of $100 \mathrm{~m} \mathrm{~s}^{-1}$ would be required to explain the extreme distortion observed. Identified drivers for such winds include (i) uneven insolation on the surface frost, with the resulting flows that maintain vapor-pressure equilibrium, and (ii) differences in albedo that lead to thermal differentials across the surface. Neither of these mechanisms would be expected to produce the high wind speeds required by the atmospheric distortion.

Immediately following the Voyager encounter, Ingersoll (1990) discussed the implications of vapor-pressure equilibrium for producing winds on Triton. Applying only mass conservation to a non-rotating body, he found that the flow velocity required to maintain vapor pressure equilibrium is only about $0.32 \mathrm{~m} \mathrm{~s}^{-1}$ (nearly three orders of magnitude less than the sonic velocity). Considering the effects of Coriolis forces and the Ekman layer, he raised this estimated wind speed to $78 \mathrm{~m} \mathrm{~s}^{-1}$, a value much closer to the magnitude of the wind speeds needed to account for the atmospheric distortion that we observed. However, Ingersoll (1990) points out that the wind speed for an outflow vortex would be limited by turbulence and the rotation speed of Triton $(16.7 \mathrm{~m}$ $\mathrm{s}^{-1}$ at the equator). The turbulence limit is highly uncertain due to uncertainty in the drag coefficient, but he concludes that wind speed, both in the Ekman layer and in the atmosphere above, is about $5 \mathrm{~m} \mathrm{~s}^{-1}$.

Recently Forget et al. (1999) discussed a 3-D general circulation model for Triton, in which they attempted to reproduce the Voyager 2 observations. However, their model is inconsistent with wind directions required to explain the observed plume drift. Ingersoll's (1990) scenario, which produced the wind directions observed by Voyager, was examined, but their model was unable to reproduce his results. No wind speeds were reported for their model.

These two works were aimed at explaining the wind speeds and directions inferred from Voyager data and do not address the issue of wind speeds approaching the sonic velocity. So 
TABLE XIV

A tmospheric Pressures and Equivalent-I sothermal Temperatures

\begin{tabular}{|c|c|c|c|c|c|}
\hline Event & $\begin{array}{l}\text { Occultation } \\
\text { date }\end{array}$ & $\begin{array}{l}\text { Surface pressure } \\
\quad(\mu \mathrm{bar})\end{array}$ & $\begin{array}{l}\text { Equivalent-isothermal pressure } \\
\text { at } 1400 \mathrm{~km}(\mu \text { bar })\end{array}$ & $\begin{array}{l}\text { Equivalent-isothermal } \\
\text { temperature }(\mathrm{K})\end{array}$ & References \\
\hline Voyager & $1989-08-25$ & $14 \pm 1$ & - & - & Tyler et al. (1989); Gurrola (1995) \\
\hline Tr60 & $1993-07-10$ & - & $1.8_{-1.2}^{+7.9 b}$ & $57.7_{-7.6}^{+8.2 b}$ & Olkin et al.(1997) \\
\hline $\operatorname{Tr} 148$ & 1995-08-14 & - & $1.49 \pm 0.14^{c}$ & $46.7 \pm 1.4^{c}$ & Olkin et al. (1997) \\
\hline $\operatorname{Tr} 176$ & 1997-07-18 & - & $2.23 \pm 0.28$ & $43.6 \pm 3.7$ & This work (circular solution) \\
\hline $\operatorname{Tr} 176$ & 1997-07-18 & - & $2.45 \pm 0.32$ & $42.0 \pm 3.6$ & This work (elliptical solution) \\
\hline $\operatorname{Tr} 180$ & 1997-11-04 & $19.0_{-1.5}^{+1.8}$ & $2.15 \pm 0.02^{d}$ & $49.4 \pm 0.2^{d}$ & Elliot et al. (1998) \\
\hline
\end{tabular}

${ }^{a}$ Equivalent-isothermal pressure at a radius of $1400 \mathrm{~km}$ from the center of Triton.

${ }^{b}$ Error bars are large because this was a single-chord event with no central flash.

${ }^{c}$ Weighted average of the values given in Table IX of Olkin et al. (1997).

${ }^{d}$ Elliot et al. (1998) published values for a model with a thermal gradient; entered here are the equivalent-isothermal values for an isothermal model fit to the same data.

it appears that more theoretical effort is needed to determine whether high-speed winds could be produced in Triton's atmosphere without violating other observational constraints. For example, the wind speed in a "winter" inflow vortex in the Northern polar region would not be subject to the upper limit of Triton's rotational speed, and wind speeds significantly greater than those considered by Ingersoll (1990) may be possible.

\section{Pressure}

Triton's atmosphere has been reported to have undergone an increase in pressure (Elliot et al. 1998, 2000, Sicardy et al. 1998) since the time of the Voyager encounter in August 1989, when its surface pressure was measured to be $14 \pm 1 \mu$ bar with an occultation of the spacecraft radio signals (Tyler et al. 1989, Gurrola 1995). For an $\mathrm{N}_{2}$ atmosphere in vapor-pressure equilibrium with surface frost, this pressure corresponds to a surface-frost temperature of $37.5 \pm 0.1 \mathrm{~K}$. With this occultation we did not measure the surface pressure, but we can compare the pressure at a radius of $1400 \mathrm{~km}$ (48-km altitude) with that derived from other stellar occultations.

In Table XIV we have entered the equivalent-isothermal pressures derived from the four Triton occultations observed since 1993. These are weighted means of the values from all stations in the case of this event and the Tr148 occultation. An equivalent-isothermal solution for the Tr180 light curve was not published by Elliot et al. (1998), so we have carried out an equivalent-isothermal solution for that data set and entered the results in Table XIV. Their published fit included a thermal gradient as a free parameter, for which the pressure at $1400 \mathrm{~km}$ is $2.30 \pm 0.03 \mu \mathrm{bar}$, while the equivalent-isothermal pressure that we have entered in Table XIV is $2.15 \pm 0.02 \mu$ bar. The pressure at $1400 \mathrm{~km}$ determined from inversion of the light immersion and emersion light curves, however, is $1.8 \pm 0.1 \mu$ bar (Elliot et al. 2000). The inversion pressure should be the most accurate pressure at a radius of $1400 \mathrm{~km}$ determined from the occultation data for $\operatorname{Tr} 180$. The equivalent-isothermal pressure is about $19 \%$ higher than the inversion pressure because the thermal structure of Triton's atmosphere is not isothermal in the region probed by the stellar occultation (Elliot et al. 2000). Similar differences between the actual pressure and the equivalent-isothermal pressure are expected to exist between the other data sets, but the signal-to-noise ratios for these (including the present data set) were not great enough to allow an inversion in order to obtain the actual pressure versus altitude (as was possible for the $\operatorname{Tr} 180$ light curve). Hence we shall use the equivalent-isothermal pressures to compare occultation results.

We have plotted the equivalent-isothermal pressures at $1400 \mathrm{~km}$ for the Tr148, Tr176, and Tr180 occultations in Fig. 8. For this plot, the circular solutions were used for Tr176 to make the results directly comparable to those solutions for $\operatorname{Tr} 148$ and $\operatorname{Tr} 180$. The Tr60 pressure was omitted because it has much

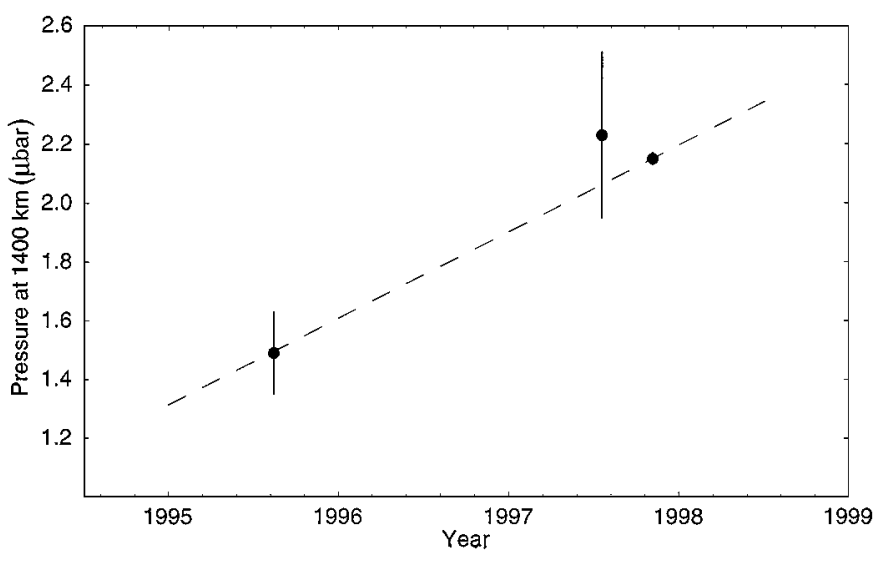

FIG. 8. Pressure at $1400 \mathrm{~km}$ versus year. The equivalent-isothermal pressures (calculated with circular solution methods) at a radius of $1400 \mathrm{~km}(48-\mathrm{km}$ altitude) determined from the $\operatorname{Tr} 148, \operatorname{Tr} 176$, and $\operatorname{Tr} 180$ occultations (given in Table XIII) have been plotted versus year. The dashed line is a weighted leastsquares fit to the three points, which shows a trend of increasing pressure of $0.29 \pm 0.04 \mu$ bar year $^{-1}$ at this level. However, extrapolating this linear trend back to the time of the Voyager encounter yields the unphysical result of an impossibly low pressure. 
greater error bars than the others. The straight line in this figure is the result of a weighted linear fit to these three points, and it has a slope of $0.29 \pm 0.04 \mu$ bar year $^{-1}$, which indicates a significant trend for an increase in this pressure during the 2.2-year interval spanned by these occultations. This rate of increase in the equivalent-isothermal pressure at $1400 \mathrm{~km}$ could not have been occurring since the time of the Voyager encounter, however, since extrapolation back to August 1989 yields an impossibly low pressure.

The equivalent-isothermal temperatures given in Table XIV exhibit a variation greater than expected from their formal errors, and this variation does not indicate a uniform trend. This may be indicating an actual change in temperature within the altitude range probed by the occultations or it may be just indicating a change in the thermal gradient in this region, since the equivalent-isothermal temperature is a combination of the actual temperature and its gradient (Elliot and Young 1992). It has been found that the thermal structure in the middle atmosphere does not agree with post-Voyager models and may be changing with time (Elliot et al. 2000). However, the thermal structure of the middle atmosphere has much less affect on the surface pressure than does the energy budget of Triton's surface (due to vapor-pressure equilibrium).

Considering the large and variable ellipticities for Triton's atmosphere exhibited by the occultation data, how certain can we be of the increasing atmospheric pressure at a radius of $1400 \mathrm{~km}$ ? Could these results be just an artifact of a variable atmospheric shape? Although we do not have a time-dependent global circu- lation model for Triton in the context of which we could interpret our occultation data, we believe that the increasing pressure at a radius of $1400 \mathrm{~km}$ is a robust result for several reasons. First, we have several light curves for both the $\operatorname{Tr} 148$ and the $\operatorname{Tr} 176$ occultations, which sampled different locations around the limb. Hence the resulting pressure averages for each event represent an average pressure around the limb of Triton (although not evenly sampled as one would like). An individual station, such as Brownsville-which has a large influence on the solution for the elliptical atmospheric figure-has only a small effect on the average pressure determined for the $\operatorname{Tr} 176$ event: the weighted average of the pressures for the circular solution (Table XII-A) yields $p_{1400}=2.13 \pm 0.30 \mu$ bar without the Brownsville results included, compared with a weighted average of $2.23 \pm 0.28 \mu$ bar for all five stations.

A second reason for believing the increase in atmospheric pressure is the consistency of this result. Both the Tr176 and the Tr180 events, which occurred just 4 months apart, show agreement between the derived pressures (Table XIV and Fig. 8). Modeling Tr176 pressure contours as an ellipse with the method described above leads to pressures for the $1400-\mathrm{km}$ isobar as given in Table XII-B only slightly different from those for the circular solutions listed in Table XII-A. Figure 9 plots the weighted mean of the $\operatorname{Tr} 176$ elliptical solutions against the circular solutions for $\operatorname{Tr} 148$ and $\operatorname{Tr} 180$, in addition to displaying the $\operatorname{Tr} 180$ inversion solution (Elliot et al. 2000), its extrapolation to the surface, and the Voyager surface pressure results (Tyler et al. 1989). For the levels of comparison, 2-4 scale heights below

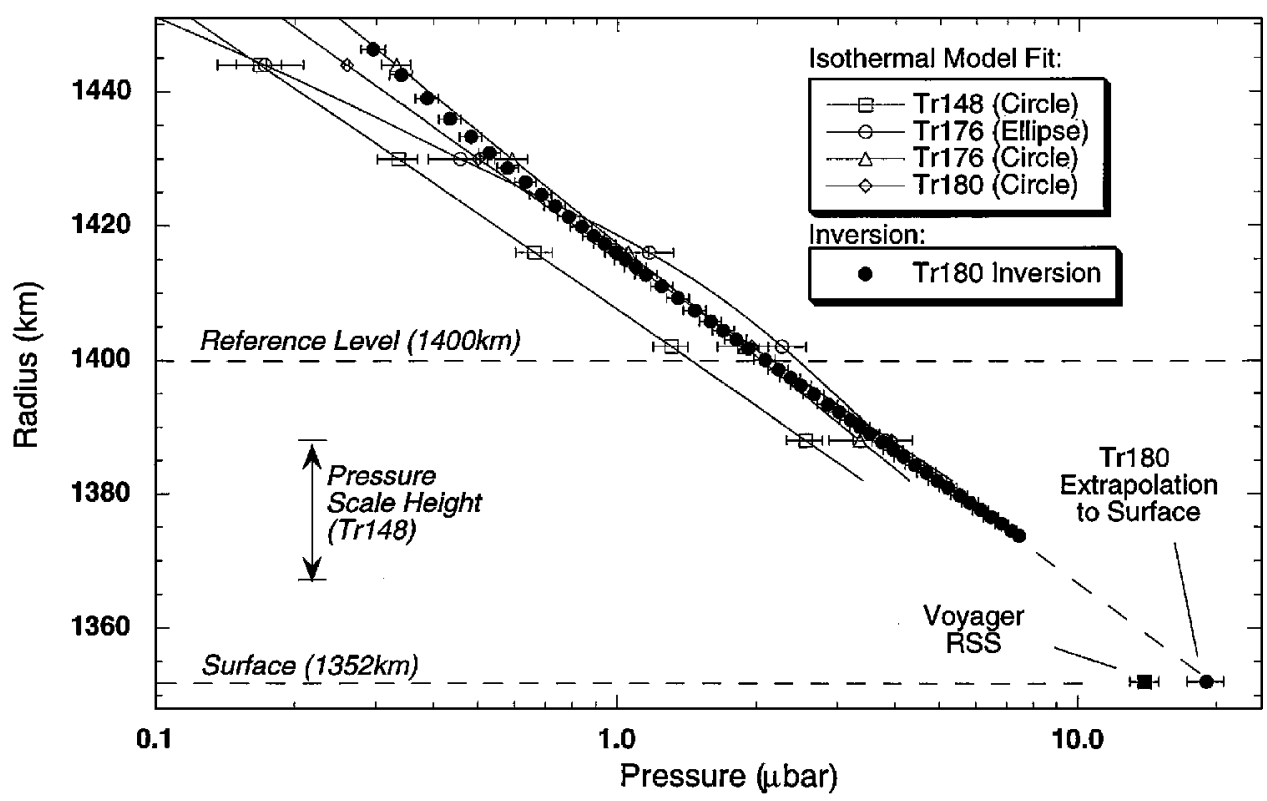

FIG . 9. Pressure versus radius on Triton. For each of the three Triton occultation data sets, pressure is plotted against radius in the atmosphere. Pressures for the circular solutions for $\operatorname{Tr} 148$, $\operatorname{Tr} 176$, and $\operatorname{Tr} 180$ are extrapolations from half-light pressure for a spherical, equivalent-isothermal atmosphere. Pressures for the Tr176 elliptical model follow the methodology described in the text. Also plotted are the pressures from direct inversion of the Tr180 light curve (Elliot et al. 2000) and their extrapolation to the surface as well as the Voyager measured surface pressure (Tyler et al. 1989, Gurrola 1995). The curvature of the Tr176 (ellipse) line is due to the elliptical extrapolation method discussed in the text. Within their error bars, the data indicate that atmospheric pressure increased in the time between Tr148 and the 1997 occultations for levels approximately two to four scale heights below half-light. 
the various half-light levels of the occultations, (approximately $1380-1420 \mathrm{~km}$ ), there is a clear increase in pressure between $\operatorname{Tr} 148$ and the 1997 curves. The Tr176 elliptical solution matches the $\operatorname{Tr} 180$ (circular) solution within its error bars for this entire region. A complete analysis would recompute the $\operatorname{Tr} 148$ best fitting isothermal models of (Olkin et al. 1997), and the $\operatorname{Tr} 180$ equivalent isothermal pressure given in Table XIII, by allowing ellipticity in the refractivity contours, but this approach is beyond our current scope.

Finally, lest one be concerned that the conclusion of an increasing atmospheric pressure is wholly dependent on comparisons with the Tr148 results (Olkin et al. 1997), the inversion results of the Tr180 data set (Elliot et al. 2000) show an increase in surface pressure when compared with Voyager, as shown in Table XIV and Fig. 9. The Tr180 data set has an extremely high signal-to-noise ratio (about 630 compared with values for this data set ranging between 13.2 and 34.1 in Table IX-A), and inversions for the temperature, number density, and pressure reached a lower altitude limit of about $\sim 20 \mathrm{~km}$ above the surface, allowing extrapolation of the pressure to the surface to be carried out with minimal uncertainty.

Since Triton's atmospheric pressure is determined by vaporpressure equilibrium with surface frosts, the noted pressure change can be compared with predictions from models for the seasonal transport of these frosts. In a survey of models based on the thermal inertia of the surface of Triton and the migration of frosts, those models with high thermal inertias predict an increasing pressure during the 1990s (Hansen and Paige 1992, Spencer and Moore 1992). One of the largest predicted increases is a 12- $\mu$ bar increase between the time of the Voyager encounter in 1989 and the time of the $\operatorname{Tr} 180$ occultation (Model L of Spencer and Moore 1992). This compares with the observed surface-pressure increase of $5 \pm 2 \mu$ bar between the Voyager measurements and the Tr180 occultation (Table XIV). Models in this class are consistent with our data. On the other hand, models with a low or zero thermal inertia lead to possible atmospheric collapse during this period (Spencer 1990) - the opposite of what we observed. A typical model of this type is Model J of Spencer and Moore (1992), which predicts a collapse down to a surface pressure of 2-3 $\mu$ bar.

Large changes in Triton's spectral and photometric properties have recently been reported (Buratti et al. 1994, 1999). These may be indicative of changes in the albedo and emissivity of the surface frosts that could be occurring during the seasonal insolation cycle, which, by raising the mean surfacefrost temperature, would cause a corresponding increase in surface pressure. This and other mechanisms are discussed by Elliot et al. (1998). Finally, the increasing pressure is consistent with proposed Koyaanismuuyaw models (Moore and Spencer 1990, Spencer 1990, Spencer and Moore 1992) that include a permanent polar frost cap (as opposed to strictly seasonal frost coverage), in which case the increasing pressure would be driven by the changing fraction of insolation over this permanent feature (Elliot et al. 1998).

\section{CONCLUSIONS}

Although we did not succeed in our goals of recording several light curves within the central flash in order to establish the shape of Triton's atmosphere at an altitude of $\sim 20 \mathrm{~km}$, our results confirm the conclusion of Elliot et al. (1998) that the pressure of Triton's atmosphere at $1400 \mathrm{~km}$ has been increasing-most likely due to a slight warming of the surface that has released more $\mathrm{N}_{2}$ in to the atmosphere. Also, as in past occultations by Triton, we find that the temperatures and pressures derived from light curves recorded at different stations are the same (within their uncertainties). Hence our results are consistent with no variation-for different locations on Triton-in atmospheric temperatures and pressures at an altitude of about $48 \mathrm{~km}$. This uniformity is consistent with the atmosphere being in vapor-pressure equilibrium with $\mathrm{N}_{2}$ surface frost.

We find a significant deviation from a circular shape of the half-light surface of Triton's atmosphere, which we have modeled as an ellipse that has a semimajor axis of $1463 \pm 10 \mathrm{~km}$ (in Triton's shadow plane) and an ellipticity of $0.040 \pm 0.003$. This elliptical solution should not be considered a definitive result for the global shape of Triton's atmosphere-because the data are not nearly dense enough to establish its global shape. But the much better fit of the elliptical model compared with the circular model does indicate that Triton's atmosphere is distorted from a spherical shape-most likely caused by high-speed winds. This result leaves us faced with the problem of understanding how the isobar at half-light can be so distorted from a circular shape, yet, just over $100 \mathrm{~km}$ below, the isobar at the surface of Triton must conform to a circle, as would be required by the vapor-pressure equilibrium of $\mathrm{N}_{2}$ gas with its surface frost. Further, more detailed observations of Triton's atmospheric shape as a function of time may help to elucidate the origin of such unexpectedly large (and variable) distortion from a sphere.

Our occultation prediction had a cross-track error of only 12 mas. This is the angle subtended by the radius of an $80-\mathrm{km}$ diameter Trojan asteroid at a distance of $4 \mathrm{AU}$, the radius of a 500-km diameter Centaur at a distance of $29 \mathrm{AU}$, and the radius of an $800-\mathrm{km}$ diameter Kuiper belt object (KBO) at a distance of $40 \mathrm{AU}$. However, predicting occultations of stars by Trojans, Centaurs, and KBOs present the additional challenges of (i) performing accurate astrometry on much fainter bodies and (ii) accurate extrapolation of orbits less well known than that of Triton. From multi-chord observations of occultations by these bodies we can establish their diameters, which can be used to calibrate thermal models for those for which we have photometry at thermal wavelengths. With further improvements to our astrometric methods accurate predictions for these smaller bodies should become achievable in the future.

\section{ACK NOW LED G MENTS}

Many people helped us with the work needed for the prediction and observation of this occultation. We thank Oscar Saa, Arturo Gomez, and their colleagues for assistance in setting up for our astrometric observations at CTIO. Mary Agner 
worked with the MIT team in obtaining the CTIO observations and helped with the astrometric reduction leading to our prediction. Amanda Bosh and Amanda Sickafoose carried out the astrometric observations at USNO. John Stansberry worked on the astrometric reductions at Lowell, and Otto Franz supplied his invaluable judgment in the interpretation of the FGS data. The Williams team appreciates help from Ken Mottram, Terry Murphy, and Brad Carter of the Centre for Astronomy and Atmospheric Research, University of Southern Queensland. The LPL team was greatly assisted by Professors Arnulfo Mar and Mario Diaz of the University of Texas at Brownsville, and we thank Professor Diaz and his family for allowing their residence to be used as the site for the LPL observations. Peter Birch assisted us with the Mount Stromlo observations. We greatly appreciate permission to use the Bundaberg, Ducabrook, and Lochington data in advance of publication from Bruno Sicardy and Wolfgang Beisker. The observers on their teams were E. Hummel, of the IOTA-European Section, Hanover, Germany; P. Anderson, L. Ball, S. Hutcheon, M. Moy, and G. Nielsen of the Astronomical Association of Queensland, St. Lucia, Queensland, Australia; and I. Pink and R. Walters, of the Bundaberg Astronomical Society Inc. C. Wunsch, A. Plumb, and A. Ingersoll provided helpful discussions and advice. Also we are grateful to an anonymous referee for many insightful comments and suggestions that improved the manuscript. This work was partially supported by Grant 5976-97 from the National Geographic Committee for Research and Exploration, by Grant GO-07489 from the Space Telescope Science Institute, by Grants ATM9707907 and ATM9812408 from NSF's Atmospheric Sciences Program, and by Grants NAG5-4214, NAG5-4195, and NAG5-3940 from NASA's Planetary Astronomy Program.

\section{REFERENCES}

Anderson, J. D., S. W. Asmar, J. K. Campbell, R. A. Jacobson, T. P. Kushner, E. R. Kurinski, E. L. Lau, and D. D. Morabito 1992. Gravitational Parameters for Neptune and Triton. Neptune and Triton Conference Proceedings, Tucson, AZ, p. 1.

Bevington, P. R., and D. K. Robinson 1992. Data Reduction and Error Analysis for the Physical Sciences. McGraw-Hill, New York.

Bosh, A. S., L. A. Young, J. L. Elliot, H. B. Hammel, and R. L. Baron 1992. Photometric variability of Charon at $2.2 \mu \mathrm{m}$. Icarus 95, 319-324.

Buie, M. W., and S. J. Bus 1992. Physical observations of (5145) Pholus. Icarus 100, 288-294.

Buie, M. W., R. L. Millis, L. H. Wasserman, J. L. Elliot, S. J. Bus, E. W. Dunham, E. F. Young, W. B. Hubbard, D. M. Hunten, and W. K. Wells 1993. CCD camera occultation system. Bull. Am. Astron. Soc. 25, 1115.

Buratti, B. J., J. D. Goguen, J. Gibson, and J. Mosher 1994. Historical photometric evidence for volatile migration on Triton. Icarus 110, 303-314.

Buratti, B. J., M. D. Hicks, and R. L. Newburn 1999. Does global warming make Triton blush? Nature 397, 219-219.

Dunham, E. W. 1995. Optical instrumentation for airborne astronomy. In Proceedings of the Airborne Astronomy Symposium on the Galactic Ecosystem: From Gas to Stars to Dust (M. R. Haas, J. A. Davidson, and E. F. Erickson, Eds.), pp. 517-522. ASP, San Francisco.

Dunham, E. W., R. L. Baron, J. L. Elliot, J. V. Vallerga, J. P. Doty, and G. R. Ricker 1985. A high-speed, dual-CCD imaging photometer. Publ. Astron. Soc. Pacific 97, 1196-1204.

Dunham, E. W., S. W. McDonald, and J. L. Elliot 1991. Pluto-Charon stellar occultation candidates: 1990-1995. Astron. J. 102, 1464-1484.

Elliot, J. L., and L. A. Young 1992. Analysis of stellar occultation data for planetary atmospheres. I. Model fitting, with application to Pluto. Astron. J. 103, 991-1015.

Elliot, J. L., A. S. Bosh, M. L. Cooke, R. C. Bless, M. J. Nelson, J. W. Percival, M. J. Taylor, J. F. Dolan, E. L. Robinson, and G. W. van Citters 1993. An occultation by Saturn's rings on 1991 October 2-3 observed with the Hubble Space Telescope. Astron. J. 106, 2544-2572.
Elliot, J. L., E. W. Dunham, R. L. Baron, A. W. Watts, S. P. Kruse, W. R. Rose, and C. M. Gillespie 1989. Image quality of the Kuiper Airborne Observatory. I. Results of the first flight series. Publ. Astron. Soc. Pacific 101, 737764.

Elliot, J. L., R. G. French, E. Dunham, P. J. Gierasch, J. Veverka, C. Church, and C. Sagan 1977. Occultation of $\varepsilon$ Geminorum by Mars. II. The structure and extinction of the Martian upper atmosphere. Astrophys. J. 217, 661-679.

Elliot, J. L., H. B. Hammel, L. H. Wasserman, O. G. Franz, S. W. McDonald, M. J. Person, C. B. Olkin, E. W. Dunham, J. R. Spencer, J. A. Stansberry, M. W. Buie, J. M. Pasachoff, B. A. Babcock, and T. H. McConnochie 1998. Global warming on Triton. Nature 393, 765-767.

Elliot, J. L., J. A. Stansberry, C. B. Olkin, M. A. Agner, and M. E. Davies 1997. Triton's distorted atmosphere. Science 278, 436-439.

Elliot, J. L., D. F. Strobel, X. Zhu, J. A. Stansberry, L. H. Wasserman, and O. G. Franz 2000. The thermal structure of Triton's middle atmosphere. Icarus $\mathbf{1 4 3}$, 425-428.

Forget, F., N. Decamp, and F. Hourdin 1999. A 3-D General Circulation Model of Triton's Atmosphere and Surface. Pluto and Triton: Comparisons and Evolution over Time. Lowell Observatory, Flagstaff, AZ.

Gurrola, E. M. 1995. Interpretation of Radar Data from the Icy Galilean Satellites and Triton. Ph.D. thesis, Stanford University, Palo Alto.

Hansen, C. J., and D. A. Paige 1992. A thermal model for the seasonal nitrogen cycle on Triton. Icarus 99, 273-288.

Hansen, C. J., A. S. McEwen, A. P. Ingersoll, and R. J. Terrile 1990. Surface and airborne evidence for plumes and winds in Triton. Science 250, 421424.

Hubbard, W. B., B. Sicardy, R. Miles, A. J. Hollis, R. W. Forrest, I. K. M. Nicolson, G. Appleby, W. Beisker, C. Bittner, H.-J. Bode, M. Bruns, H. Denzau, M. Nezel, E. Riedel, H. Struckmann, J. E. Arlot, F. Roques, F. Sévre, W. Thuillot, M. Hoffman, E. H. Geyer, C. Buil, F. Colas, J. Lecacheux, A. Klotz, E. Thouvenot, J. L. Vidal, E. Carreria, F. Rossi, C. Blanco, S. Cristaldi, Y. Nevo, H. J. Reitsema, N. Brosch, K. Cernis, K. Zdanavicius, L. H. Wasserman, D. M. Hunten, D. Gautier, E. Lellouch, R. V. Yelle, B. Rizk, F. M. Flasar, C. C. Porco, D. Toublanc, and G. Corugedo 1993. The occultation of $28 \mathrm{Sgr}$ by Titan. Astron. Astrophys. 269, 541-563.

Ingersoll, A. P. 1990. Dynamics of Triton's atmosphere. Nature 344, 315-317.

McDonald, S. W., and J. L. Elliot 1995. Triton stellar occultation candidates: 1995-1999. Astron. J. 109, 1352-1362.

Moore, J. M., and J. R. Spencer 1990. Koyaanismuuyaw: The hypothesis of a perennially dichotomous Triton. Geophys. Res. Lett. 17, 1757-1760.

Olkin, C. B., J. L. Elliot, H. B. Hammel, A. R. Cooray, S. W. McDonald, J. A. Foust, A. S. Bosh, M. W. Buie, R. L. Millis, L. H. Wasserman, E. W. Dunham, J. McDonald, L. A. Young, R. Howell, W. B. Hubbard, R. Hill, R. L. Marcialis, J. S. McDonald, D. M. Rank, J. C. Holbrook, and H. Reitsema 1997. The thermal structure of Triton's atmosphere: Results from the 1993 and 1995 occultations. Icarus 129, 178-201.

Peck, E. R., and B. N. Khanna 1966. Dispersion of nitrogen. J. Opt. Soc. Am. 56, 1059-1063.

Seidelmann, P. K. 1992. Explanatory Supplement to the Astronomical Almanac. University Science Books, Mill Valley.

Sicardy, B., O. Mousis, W. Beisker, E. Hummel, W. B. Hubbard, R. Hill, H. J. Reitsema, P. Anderson, L. Ball, B. Downs, S. Hutcheon, M. Moy, G. Nielsen, I. Pink, and R. Walters 1998. Structure of Triton's atmosphere from the occultation of Tr176. Bull. Am. Astron. Soc. 30, 1107.

Smart, W. M. 1977. Textbook on Spherical Astronomy. Cambridge Univ. Press, Cambridge.

Smith, B. A., L. A. Soderblom, D. Banfield, C. Barnet, A. T. Basilevksy, R. F. Beebe, K. Bollinger, J. M. Boyce, A. Brahic, G. A. Briggs, R. H. Brown, C. Chyba, S. A. Collins, T. Colvin, A. F. Cook II, D. Crisp, S. K. Croft, D. Cruikshank, J. N. Cuzzi, G. E. Danielson, M. E. Davies, E. De Jong, L. Dones, D. Godfrey, J. Goguen, I. Grenier, V. R. Haemmerle, H. Hammel, C. J. Hansen, 
C. P. Helfenstein, C. Howell, G. E. Hunt, A. P. Ingersoll, T. V. Johnson, J. Kargel, R. Kirk, D. I. Kuehn, S. Limaye, H. Masursky, A. McEwen, D. Morrison, T. Owen, W. Owen, J. B. Pollack, C. C. Porco, K. Rages, P. Rogers, D. Rudy, C. Sagan, J. Schwartz, E. M. Shoemaker, M. Showalter, B. Sicardy, D. Simonelli, J. Spencer, L. A. Sromovsky, C. Stoker, R. G. Strom, V. E. Suomi, S. P. Synott, R. J. Terrile, P. Thomas, W. R. Thompson, A. Verbiscer, and J. Veverka 1989. Voyager 2 at Neptune: Imaging science results. Science 246, 1422-1449.

Soderblom, L. A., S. W. Kieffer, T. L. Becker, R. H. Brown, A. F. Cook II, C. J. Hansen, T. V. Johnson, R. L. Kirk, and E. M. Shoemaker 1990. Triton's geyser-like plumes: Discovery and basic characterization. Science 250, 410415.

Spencer, J. R. 1990. Nitrogen frost migration on Triton: A historical model. Geophys. Res. Lett. 17, 1769-1772.

Spencer, J. R., and J. M. Moore 1992. The influence of thermal inertia on temperatures and frost stability on Triton. Icarus 99, 261-272.
Stetson, P. B. 1987. DAOPHOT: A computer program for crowded-field stellar photometry. Publ. Astron. Soc. Pacific 99, 191-222.

Stone, R. C., D. G. Monet, A. K. B. Monet, R. L. Walker, H. D. Ables, A. R. Bird, and F. H. Harris 1996. The Flagstaff astrometric scanning transit telescope (FASTT) and star positions determined in the extragalactic reference frame. Astron. J. 111, 1721-1740.

Tody, D. 1986. The IRAF data reduction and analysis system. Proc. SPIE 627, 733-748.

Tyler, G. L., D. N. Sweetnam, J. D. Anderson, S. E. Borutzki, J. K. Campbell, V. R. Eshleman, D. L. Gresh, E. M. Gurrola, D. P. Hinson, N. Kawashima, E. R. Kursinski, G. S. Levy, G. F. Lindal, J. R. Lyons, E. A. Marouf, P. A. Rosen, R. A. Simpson, and G. E. Wood 1989. Voyager radio science observations of Neptune and Triton. Science 246, 1466-1473.

Zharkov, V. N., V. V. Leontjev, and A. V. Kozenko 1985. Models, figures, and gravitational moments of the Galilean satellites of Jupiter and the icy satellites of Saturn. Icarus 61, 92-100. 